Florida International University

FIU Digital Commons

FIU Electronic Theses and Dissertations

University Graduate School

7-29-2004

\title{
Acculturation and Hispanic identity/ethnicity as a predictor of change on the brief situational confidence questionnaire
}

Simy Benchimol

Florida International University

DOI: $10.25148 /$ etd.FI14050493

Follow this and additional works at: https://digitalcommons.fiu.edu/etd

Part of the Psychiatry and Psychology Commons

\section{Recommended Citation}

Benchimol, Simy, "Acculturation and Hispanic identity/ethnicity as a predictor of change on the brief situational confidence questionnaire" (2004). FIU Electronic Theses and Dissertations. 1494.

https://digitalcommons.fiu.edu/etd/1494 
FLORIDA INTERNATIONAL UNIVERSITY

Miami, Florida

ACCULTURATION AND HISPANIC IDENTITY/ETHNICITY AS A PREDICTOR

OF CHANGE ON THE BRIEF SITUATIONAL CONFIDENCE QUESTIONNAIRE

A thesis submitted in partial fulfillment of the

requirements for the degree of

MASTER OF SCIENCE

in

PSYCHOLOGY

by

Simy Benchimol

2004 
To: Dean R. Bruce Dunlap

College of Arts and Sciences

This thesis, written by Simy Benchimol, and entitled Acculturation and Hispanic Identity/Ethnicity as a Predictor of Change on the Brief Situational Confidence Questionnaire, having been approved in respect to style and intellectual content, is referred to you for judgment.

We have read this thesis and recommend that it be approved.

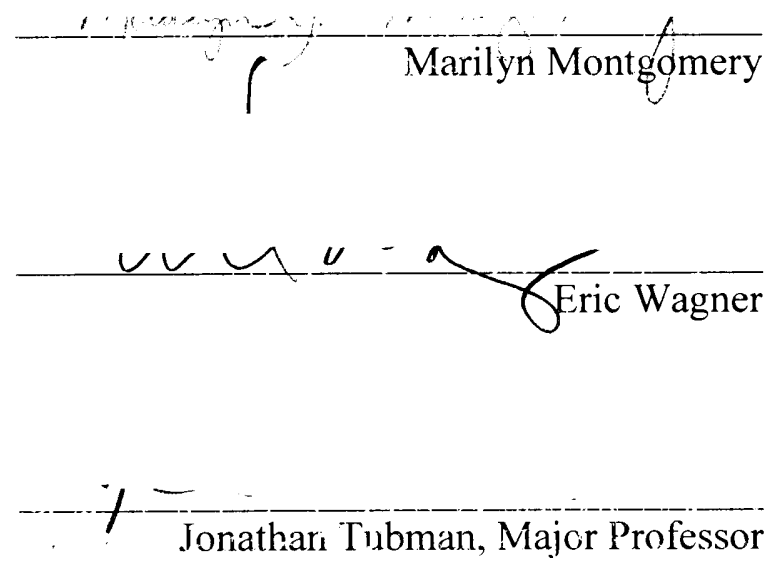

Date of Defense: July 29, 2004

The thesis of Simy Benchimol is approved.

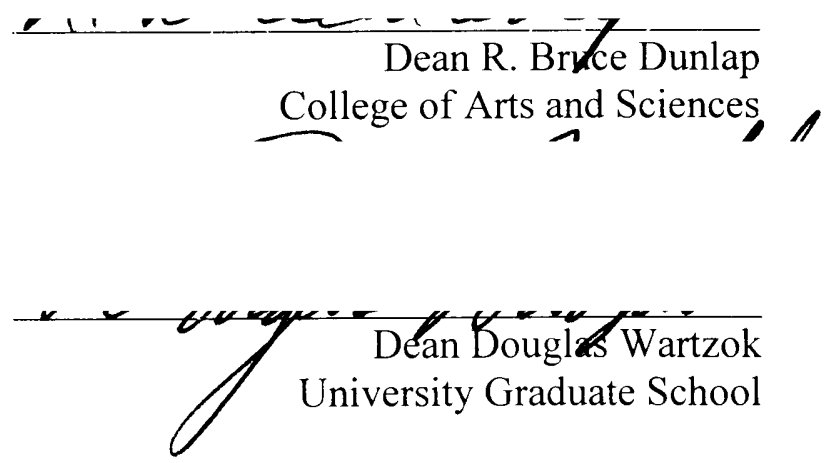

Florida International University, 2004 
(C) Copyright 2004 by Simy Benchimol

All rights reserved. 


\section{DEDICATION}

I dedicate this thesis to my family; my father Isaac, my brother Daniel, and especially to my mother Elizabeth. Her love, guidance, unending support and sacrifice have made it possible for me to get this far. 


\section{ACKNOWLEDGMENTS}

This thesis would not have been possible without the support and guidance of my committee chair and advisor, Dr. Jonathan Tubman. Thank you for all your patience and caring support, and for allowing me to continuously grow as a person and professional. I would also like to thank my thesis committee members, Drs. Marilyn Montgomery and Eric Wagner for sharing their knowledge and providing me with guidance throughout this process. You have both played a helpful role in the progress of this thesis.

I would also like to thank Dr. Andres Gil, Lynn Hernandez, and Jessica Kissane, for guiding me through the world of analysis and everyone at the Community Based Intervention Research Group (C-BIRG). To Rachel Breslof, Jessica Concepcion, Eyra Gayol, Roxanne Munoz, and Giovanna Nieves for always being there to listen to me vent and provide much needed support and distractions. Additionally, I would like to give a special acknowledgement to Nilo A. Cuervo, Jr., his unending dedication to me and his words of motivation helped make this thesis possible and for keeping me strong through it all. Words cannot express what this has meant to me. Lastly, I would like to thank G-d for guiding me, helping me achieve all my goals, and for answering all my prayers. 


\begin{abstract}
OF THE THESIS
ACCULTURATION AND HISPANIC IDENTITY/ETHNICITY AS A PREDICTOR

OF CHANGE ON THE BRIEF SITUATIONAL CONFIDENCE QUESTIONNAIRE

by
\end{abstract}

Simy Benchimol

Florida International University, 2004

Miami, Florida

Professor Jonathan Tubman, Major Professor

The purpose of this study was to investigate whether level of acculturation among Hispanic adolescent males $(n=174)$ influenced treatment outcome in a substance abuse program, specifically on the Brief Situational Confidence Questionnaire (BSCQ) which measures relapse confidence. It was hypothesized that lower levels of acculturation were likely to be predictive of positive change, whereas higher levels of acculturation were likely to be predictive of no change or negative change. It was found that adolescents changed over time in BSCQ scores regardless of which acculturation variable was measured. Contrary to expectations, for those adolescents placed in family treatment, place of birth was not significantly associated with treatment response. However, both, U.S. and non-U.S. born adolescents demonstrated a change over time when receiving family treatment, suggesting that the family substance abuse treatment utilized in this intervention effected change over time. 
I. INTRODUCTION

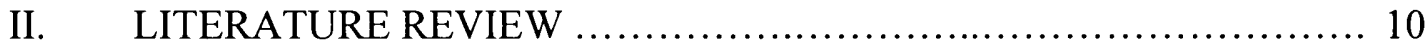

Theoretical Overview................................................ 10

Conceptual Framework ................................................................ 10

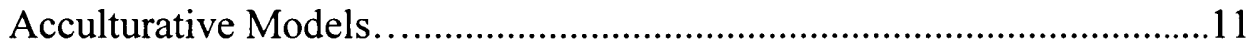

Acculturative Stress and Adjustment................................13

Relationship between Acculturation and AOD use................................. 15

Alcohol and Other Drug Use Patterns Among Hispanic/Latino

Adolescents ............................................... 16

Summary..................................................... 17

Review of Current Research on Acculturation and Therapeutic

Interventions ................................................ 18

Social and Linguistic Acculturation ...............................18

Risk and Predictive Factors ...................................... 19

Guided Self Change .............................................. 23

Relapse Prevention and Brief Situational Confidence.................. 25

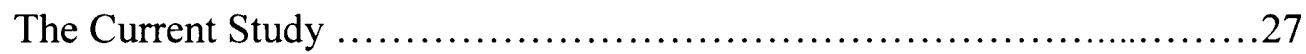

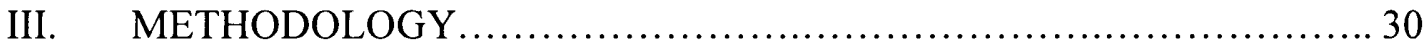

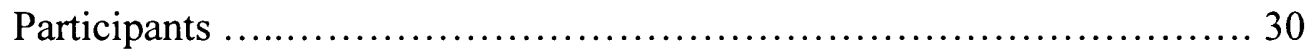

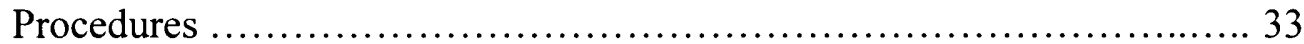

Measures................................................................. 33

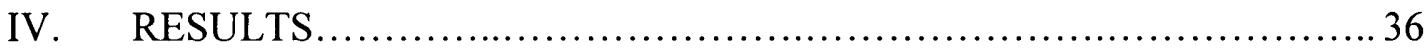

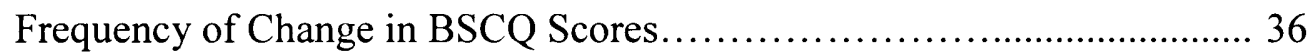

Levels of Acculturation............................................ 38

BSCQ Change Due to Acculturation Variables............................40

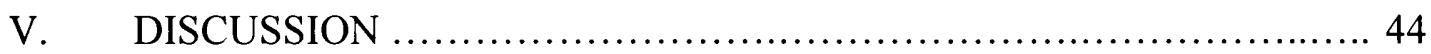

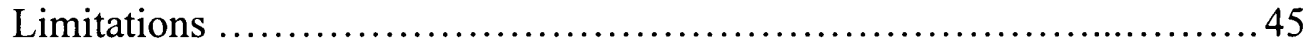

Contribution to Knowledge ..........................................46

Clinical Implications Based on Evidence................................. 48

Clinical Insight... ............................................. 49

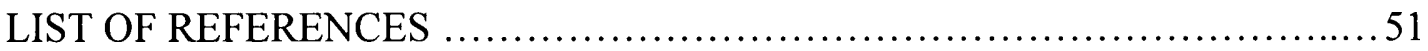

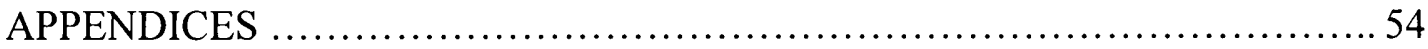




\section{LIST OF TABLES}

TABLE

PAGE

1. Hispanic Participants' Background Information ....................... 32

2. Descriptive Statistics for Study Variables ............................ 36

3. Participants' Level of Acculturation.................................. 39

4. ANCOVA for BSCQ Change Across Acculturation Variables............ 40

5. BSCQ Score Change Across Nativity and Family Treatment Condition........................................................... 41

6. ANOVA for BSCQ Change Across Place of Birth.................... 42 


\section{CHAPTER 1}

\section{INTRODUCTION}

\section{Adolescent Substance Abuse}

Adolescence is a time of exploration and experimentation. As children begin to mature into young adults, they start to become more curious about the world that is developing around them. Not only are they maturing biologically, but cognitive, social, and psychological changes also start to occur. They now begin to see and understand how they can impact their rapidly changing social world, i.e., the influence they have on the people around them, including peers and parents. Changes in social definition also occur at adolescence. Adolescents are given certain privileges and rights in society typically reserved for adults which leads to increased expectations for self-management, personal responsibility, and social participation (Steinberg, 1999). At this age, adolescents are also more likely to engage in normative, exploratory risk behaviors (Quadrel, Fischoff, \& Davis, 1993). Although the current literature states that adolescents, like adults, are no more likely to view themselves as invulnerable in risky situations, maldaptive thought processes can alter effective decision making (Moore \& Gullone, 1996). Where an adult may make decisions based more on the likelihood of health risks, adolescents often make decisions that are more heavily driven by social influences. As a result of their curiosity and the high values placed on peer relationships, adolescents often find themselves attracted to experimentation with alcohol and other drug (AOD) use.

Experimentation with alcohol, cigarettes, and illicit drugs during early

adolescence is an important marker of future persistent use (Vega, Zimmerman, Warheit, 
Apospori, \& Gil, 1993). The risk for experimental substance use is higher in this age group than it is in any other. In fact, the age group at highest risk for developing a substance use problem is the one including adolescents between the ages of 15 and 19 years old (Chatlos, 1996.) Studies have also found that children and adolescents who start experimenting with AOD use earlier in adolescence are at higher risk for developing a substance use problem (Kandel, 1997).

Therefore, adolescent substance use and abuse is a significant social problem with serious short and long-term consequences for the health of the nation's youth. In 1997, more than $54 \%$ of high school seniors and more than $29 \%$ of eighth grade students had used marijuana at least once (Gullotta, Adams, \& Montemayor, 1997). According to the latest (2003) statistic from the Monitoring the Future study, $17.5 \%$ of $8^{\text {th }}$ graders, $36.4 \%$ of $10^{\text {th }}$ graders, and $46.1 \%$ of $12^{\text {th }}$ graders have used marijuana. In terms of alcohol consumption, $45.6 \%$ of $8^{\text {th }}$ graders, $66 \%$ of $10^{\text {th }}$ graders, and $76.6 \%$ of $12^{\text {th }}$ graders have used alcohol. Nationally, approximately 3 million adolescents exhibit symptoms of alcohol or drug abuse and almost $10 \%$ of older adolescents meet diagnostic criteria for an alcohol use disorder (Tweed, 1998; De Bellis, Clark, Beers, Soloff, Boring, Hall, Kersh, \& Keshavan, 2000.)

What is most disturbing is that adolescents between the ages of 12 and 17 years who exhibit no other problem behaviors and who have experimented with cigarettes, marijuana, and alcohol are 17 times more likely to experiment with heroin, cocaine, or LSD than those who have not used drugs (Gullotta, Adams, \& Montemayor, 1994). Researchers (e.g., Brunswick, 1989; Millman \& Beeder, 1994) have suggested that certain drugs such as inhalants and marijuana serve as an introduction to the future, 
possibly persistent abuse of other illicit drugs. Hogan (2000) suggested that there are five basic reasons why adolescents use or abuse substances: to feel grown up; to take risks to rebel against authority; to fit into a specific, desired peer group; to relax and feel good; and, to satisfy their curiosity about the effects of drugs.

\section{Hispanic Adolescents}

Statistics regarding substance abuse are available to adolescents in general, but it is also important to examine substance abuse statistics for specific cultural and racial groups, and in particular, Hispanics. The term Hispanic or Latino/a is used to describe individuals of Cuban, Mexican, Puerto Rican, South and Central American ancestry, regardless of race. In the state of Florida, at the time of the last census (2000), Hispanic/Latinos accounted for $16.8 \%$ of the general population and $57.3 \%$ of the population for Miami-Dade County. Yet, this population remains underresearched and underserved, especially with regard to adolescent substance abuse. Many Hispanic/Latinos leave their native countries due to war, political turmoil, or to seek better economic futures for themselves and their children. The experiences of preparing for separation from their native lands are often filled with emotional distress, financial difficulties, and social disruption and these experiences have important implications for the short- and long-term adjustment of immigrants (Vega \& Gil, 1999).

When children and adolescents experiencing critical developmental transitions also experience the immigration process, psychological adjustment can be impacted. Children who migrate when they are in middle to late adolescence will often have very different socialization experiences than those experienced by children migrating at earlier ages (Vega \& Gil, 1999). Older children have developed a better sense of identity, having 
grown up within a consistent cultural or social context, while younger immigrant or second generation children are often reared with significant family-level cultural and social conflicts or contradictory social norms and customs compared to traditional family values. Socialization experiences regarding the use of alcohol, tobacco, and other drugs can be complex for immigrant youth.

Acculturation and Substance Abuse

Acculturation typically refers to the process of adjustment that an immigrant experiences as he or she exits a native culture and starts to adopt cultural beliefs, norms, values, customs, behaviors and lifestyles of a new, and usually dominant, host culture (de la Rosa, Vega, \& Radisch, 2000). It has been proposed that acculturative stress can occur as a result of the acculturation process, and this cumulative stress involves issues such as language problems, perceived discrimination, perceived cultural incompabilties, commitment or lack thereof to culturally prescribed protective values/behaviors such as familialism and cultural pride (Gil, Vega, \& Dimas, 1994). Acculturation may also include significant family stressors involving the immigrant parental resocialization regarding contemporary U.S. teen culture, including AOD use, the dangers associated with AOD use, as well as the norms and social networks that support access to regular AOD consumption.

Many immigrant families experience significant economic hardships and they may live in lower class neighborhoods with inadequate schools, high levels of exposure to gang activity and drug use, substandard employment opportunities, and multiple forms of criminal activity. Hispanic families living in similar social contexts play important roles in the development of and the shaping of the acculturation process. Acculturation 
into the mainstream, dominant culture is a key factor in the weakening or modification of traditional family values and Hispanic familialism, which may be accompanied by signifcant stress on the family system. Vega, Gil, and Wagner (1998) suggest that Hispanic adolescents who are raised with more economic resources and who face less prejudice and discrimination than their less advantaged counterparts are less likely to experience social and personal adjustment difficulties. The absence of these difficulties and stressors are likely to protect against the development of deviant behaviors, including substance abuse.

It is only recently that researchers have investigated intensively the issue of acculturation and its significance for the development of substance use and abuse among adolescents. Hispanic adolescents are a population at higher risk for substance use due to acculturative stresses that they experience after moving to a new host country, stresses involving conflicts between the protective influences of customs and traditions of their family and the social influences of new peers and more permissive social norms. Existing data, however, on adolescent substance abuse has only recently begun to consider key social and ethnic differences in American society, and the processes that are unique to the development of Hispanic adolescents and drug use patterns (Vega \& Gil, 1999). Studies have been conducted proposing that acculturative stress is different for those Hispanic adolescents who immigrate versus those who are born in the U.S. (Gil, Wagner, \& Vega, 2000; Hovey \& King, 1996; Vega \& Gil, 1999; Vega, Gil, \& Wagner, 1998; Vega, Gil, \& Zimmerman, 1993; Warheit, Biafora, \& Zimmerman et al., 1996). These differences in stresses experienced suggest that level of acculturation is a key risk factor for the development of problem patterns of substance use. 
Foreign-born Hispanics are faced with multiple challenges, such as social transplantation, cultural changes, and acquisition of a new language, whereas native-born Hispanics grow up within a social environment where traditional Hispanic family values are may be perceived as old-fashioned by adolescents, which leads to familial stress. Adolescents who are native born may be less capable of handling situations of discrimination, prejudice, gang involvement, and subsequent substance abuse due if they lack belief in, and integration of, traditional Hispanic family values which are thought to serve a protective function (Vega \& Gil, 1999). Similar studies have found that both native and foreign born Hispanics are more likely to experience mental health problems the more acculturated they are, compared to their less acculturated foreign born counterparts (de la Rosa, Vega, Radisch, 2000; Gil, Vega, \& Dimas, 1994, Vega \& Gil, 1999). Vega, Gil, and Wagner (1998) have also found that Hispanics in Florida (mostly of Cuban ancestry) who had lower levels of acculturation, reported lower levels of substance abuse than those who were highly acculturated or considered themselves to be bicultural. Bicultural adolescents are those who have integrated successfully into American society and yet they maintain their original or their parents' cultural values. Other findings (Gil, Vega, \& Dimas, 1994), however, suggest that those adolescents who are truly bicultural may use the skills that they have learned as a protective factor against substance abuse, since traditional Hispanic values discourage the use and abuse of illicit substances.

Therefore, in terms of levels of acculturation, there are some conflicting results from contemporary research as to which is more or less maladaptive. Rogler et al. (1991) has proposed that there are in fact three different relationships between acculturation- 
related variables and social maladaption or psychopathology: positive, negative, and curvilinear. Positive developmental outcomes are more likely to occur when individuals who are highly acculturated experience lower levels of stress. Individuals with low levels of acculturation who experience low levels of stress will tend to have better mental health and psychosocial adjustment. A curvilinear relationship between acculturation and mental health outcomes occurs when a bicultural individual experiences better psychological outcomes due to their knowledge of, and participation, in the host culture, while maintaining positive and protective factors from their traditional culture.

Therefore, traditional models of, and interventions for, adolescent substance abuse may be less applicable to Hispanic/Latino populations due to the unique experiences of this population. Substance abuse researchers and therapists also should be aware of significant cultural differences within Hispanic/Latin cultural groups. In addition, they should be culturally sensitive to the norms that apply to U.S.- and foreign-born Hispanics and their families. For example, foreign-born Hispanics are more likely to maintain traditional cultural and religious values which might lead them to reject American norms and values and view therapeutic interventions more critically.

\section{Summary}

As a result of their curiosity normative exploratory risk behaviors, adolescents often find themselves involved in AOD use. Experimentation with alcohol, cigarettes, and illicit drugs during early adolescence is an important risk factor for future, persistent patterns of use. The risk for experimental substance use is higher within this age group than it is for any other age group. For a variety of reasons, subgroups of Hispanic adolescents are at higher risk for substance use initiation and socially deviant behavior, 
and yet this population remains underresearched and underserved. Hispanics who move from their country of origin to the United States normally experience significant acculturation processes, which can be very stressful. Acculturation normally refers to the process of adjustment that a person experiences as they exit their native culture and adopt the cultural beliefs, norms, values, customs, behaviors and lifestyles of the new host and the dominant culture.

Foreign-born Hispanics are faced with significant challenges, such as social transplantation, cultural changes, and the acquisition of a new language. Evidence suggests that there are significant relation between levels of acculturation and subsequent substance use and abuse. Acculturation levels among Hispanic adolescents who are foreign- versus native-born may also report significant differences in specific problem behaviors. Numerous studies have shown that acculturative stressors, both for the adolescent and within the family system, are significant predictors of substance use. The developmental stage of children or adolescents experiencing the immigration process is also critical to their future adjustment outcomes.

\section{The Proposed Study}

Taking into consideration the need for additional research with this population, the proposed study will look specifically at levels of acculturation among Hispanic male adolescents who are currently participating in a grant funded substance abuse intervention program in Miami, Florida, and its impact on adolescents' AOD use treatment response. Current program aids in the development of testing adolescents' self confidence of use and its relation to future substance use decisions especially in specific situations that are likely to impair judgment. Various levels of acculturation are a key 
mediator and will result in different levels of change in treatment response. Participants' place of birth will also be investigated to measure level of treatment response and change.

$\mathrm{H}_{1}$ : Levels of acculturation will moderate treatment impact and situational confidence in AOD use. Lower levels of acculturation are likely to be predictive of positive change, whereas higher levels of acculturation are likely to be predictive of no/negative change.

$\mathrm{H}_{2} \quad \quad$ Hispanic males who are born outside of the United States, and are less acculturated to the dominant culture, are more likely than the U.S. born and high acculturated adolescents to have a positive change in treatment when placed in family treatment condition. 


\section{CHAPTER II}

\section{LITERATURE REVIEW}

The following literature review outlines pertinent theoretical and empirical research to support the design and implementation of the proposed study. This review also provides the rationale for the current proposed research study. Four main topic areas comprise the overview of the research literature. First, the conceptual rationale and current models of acculturation will be discussed. Second, the potential impact of acculturative stress on adjustment will be reviewed. Third, the importance of level of acculturation and its status as a risk factor for AOD use among Hispanic adolescent males will be reviewed in terms of a) levels of acculturation in the dominant culture and b) acculturation differences between first and second generation Hispanics. In addition, the prevalence of AOD use and treatment utilization in Hispanic adolescent populations, specifically among male adolescents, will be reviewed. Last, research on the use and effectiveness of the Brief Situational Confidence Scale when working with this population will be discussed, as well as the Guided Self Change intervention program, and its use of a cognitive-behavioral model, designed specifically to help reduce AOD use among adolescents.

\section{Theoretical Overview}

Conceptual Framework. Albert Bandura, a social learning theorist, proposed the idea that adolescents learn how to behave by the processes of observation and modeling. Specifically, adolescents learn how to behave not simply by being reinforced and punished but by also watching and imitating those around them and in their environment (Bandura \& Walters, 1959; Steinberg, 1999). Bandura also introduced the concept of self 
efficacy. Perceived self efficacy is the term applied to a person's judgment regarding the likelihood of being able to produce specific behaviors or outcomes in particular situational contexts (Kirisci, Moss, \& Tarter, 1996). Self efficacy is an important factor to assess at when attempting to reduce current AOD use, as well as, building protective factors to encourage relapse prevention for AOD use. Low self efficacy or low levels of perceived ability to cope with high stress situations is correlated with a return to drinking behavior and smoking (Condiotte \& Lichenstein, 1981). Therefore, it is often assumed that high self efficacy is positively correlated with abstinence and that one focus of relapse prevention is the development of personal self efficacy to effectively cope with difficult situations.

Bandura (1979) states that certain criteria need to be present to increase one's confidence or self efficacy to resist substance use. Specifically, if the exposure to substance use was challenging to the client, only a moderate degree of effort was needed to experience success, little external aid was necessary, success was part of a pattern of improvement, an increase in personal control was demonstrated, and the successful performance was relevant to the client's own life situations. The adolescents participating in the current study have varying degrees of exposure to substance abuse, consequently having varying levels of improvement when receiving treatment. Clinically, when working with relapse prevention, it is helpful to work with clients to help them identify realistic situations and triggers that could lead to a relapse (Larimer, Palmer, \& Marlatt, 1999).

Acculturative Models. With the construct of acculturation becoming more of a focus of research regarding risk/protective factors for adolescent adjustment (i.e., 
substance use), there are now numerous models that have been formulated to explain its potential influence. Current theoretical models identify attitudes, subjective norms, and perceived control regarding substance use as possible determinants of substance use (Carvajal, Photiades, Evans, \& Nash, 1997). There is some debate within the research literature as how to best incorporate to the construct of acculturation into models of substance use development, intervention, and treatment. Carvajal et al. (1997) suggest Bandura's (1986) social influence model as one useful model. They suggest that as adolescents are more exposed to the dominant culture and exposed to interaction with other ethnic groups, social modeling and social influences will start to promote both adaptive and maladaptive behavioral changes and that acculturation involves multidimensional and interactive developmental processes. Their study found that, in a sample of 448 Latino adolescents, interactions with groups of peers of differing ethnicities was significantly related to participants' negative attitudes toward cigarette and marijuana use, and with friends' negative norms regarding adolescents' substance use. This suggests that key social influences moderate adolescents' substance use. In addition, perceived normative expectations against substance use by peers appear to play a key role in adolescents' future substance use.

Kaplan (1975) offers a somewhat different view. The theoretical formulation that is proposed for understanding adolescent deviant behavior is based on empirical evidence supporting relations between social deviance and parent, teacher, and peer disapproval. Adolescents who change their behaviors in order to satisfy the expectations of disapproving parents or teachers may decrease personal distress. However adolescents who don't change their behaviors will increase their levels of self-derogation and 
possibly increase levels of deviant behaviors. This formulation, when applied to Hispanic adolescents, found that there is empirical evidence to indicate that not only are problems related to acculturation processes associated with deviance, but also that high levels of family acceptance and support are correlated (i.e., as protective factors) with low levels of deviance (Rodriguez \& Weisburd, 1991).

Vega and Gil (1999) offer an alternative model that is both social and ecological in nature (see pg. 30). Their proposed model incorporates social and developmental factors within the concept of migration, and suggests that attempts to describe drug use among ethnic groups, such as Hispanics, have been inadequate because of the lack of conceptual models that address factors associated with drug use behaviors in this ethnic group. Their integrative model recognizes that drug use behaviors among Hispanic adolescents are influenced by their changing social environment, as well as key family and other social influences. Their acculturative model highlights that the importance of the context of exit, the immigrant experience, acculturation factors, segmented assimilation, acculturation stress, family stress, and drug use behavior as key elements of processes linking acculturative stress to adolescent substance abuse. This model demonstrates how levels of acculturative stress can factor to substance abuse.

Acculturative Stress and Adjustment. While, the concept of acculturation is gaining more recognition in the field of substance abuse research and measures are being developed and validated to assess levels of acculturation, there is no current measure identifying exclusively what are acculturative stressors, or the most important protective factors among children and adolescents. It has been hypothesized (Vega, Gil, \& Dimas, 1994) that second generation Hispanics who are highly acculturated are at elevated risk 
for drug use and mental health problems because they are exposed to more culturallybased conflicts, internalization of negative stereotypes, and to behavioral expectations favoring experimentation with drugs.

Acculturative stress is disproportionately experienced among less acculturated individuals because they lack the language skills, social knowledge, and personal resources to function adequately in the dominant culture, while more acculturated individuals are at a risk as well because of greater exposure to definitions of self and one's ethnic group as different, stigmatized, and having unequal access to social, educational, economic opportunities (Vega, Gil, \& Wagner, 1998) and the loss of the protective function of one's ethnicity. Mena et al. (1987) found that individuals who moved before the age of twelve experienced less acculturative stress than individuals who moved after the age of twelve. In measuring acculturation strains in sixth and seventh grade boys, Vega et al. found that $87.1 \%$ of the participants with low levels of acculturation were foreign born, and had lived in the United States for less than 2 years, while $65.1 \%$ of the sample with high levels of acculturation were born in the U.S., and had lived in the United States for approximately 6 or more years. Their study also found that Hispanic boys who were foreign born had higher exposure to acculturation strains. However, it was the sample of U.S. born boys with low levels of acculturation who had poorer outcomes, due to the effects of combined high strain and low family pride, which was widely reported in this group. U.S. born, less acculturated boys also reported higher levels of strain on self esteem as well as higher levels of strain on perceived discrimination and language conflicts. Higher levels of acculturation appeared to have 
powerful negative influences on family pride and were more common among U.S. born adolescents in this study.

Adolescents acculturate at faster rates than their parents, since they interact with American customs daily (i.e., in school, on television, in peer groups) in contrast to their parents who may encounter little intrusion of American culture upon their daily lives; especially among those adults involved in migrant, household work, or living in areas that are predominantly Hispanic (Elder et al., 2000). It has also been found that males acculturate faster than do females (Szapocznik, 1988). It has been argued that Hispanic culture acts as a general protective factor against AOD use, although it has been found that the benefits associated with particular cultures or countries of origin dissipate in subsequent generations, as adolescents become more acculturated into mainstream U.S. customs and socialized to norms that may promote alcohol and cigarette use among adolescents. Hispanic/Latino adolescents are often living in homes with older family members who are less likely to speak English, and as a result, adolescents are left without adequate support from the family to help them cope effectively with the demands of school, peer influences, and society in general.

Relationship between Acculturation and AOD use. Current research findings seem to indicate that high acculturation is associated with significantly higher drug use, especially with regard to illicit drug use (Carvajal, Photiades, Evans, \& Nash, 1997; Gil, Wagner, Vega, 2000; Vega, Gil, \& Wagner, 1998). Therefore, it is important to distinguish between immigrant and U.S. born adolescents' acculturation when studying the effects of acculturative stress risk factors on AOD use. Previous studies measuring acculturation and acculturative stress as a risk factor for drug and alcohol use would 
specifically sample and assess adults, and only recently has available research investigated this risk factor in the onset and maintenance of adolescent drug and alcohol use. Research has shown that more acculturated women have higher rates of tobacco use than less acculturated women and less acculturated men have higher rates of tobacco use than more acculturated men (Marin, Perez-Stable, \& Marin, 1989; Sabogal, OteroSabogal, Perez-Stable, Marin, et al., 1989). Acculturation level has also been linked to alcohol related behaviors in Hispanic adults (Burnam, Hough, Karno, Escobar, \& Telles, 1987). Therefore, acculturation may be an important risk factor for substance use among Hispanic youth. Vega, Gil, \& Wagner (1998) examined the direct associations between acculturation and adolescent marijuana use and found that acculturative stress contributed to low self-esteem, and both factors contributed as mediators to later adolescent substance use.

\section{Alcohol and Other Drug Use Patterns Among Hispanic/Latino Adolescents.}

Research has shown that experimentation with alcohol, cigarettes, and illicit drugs during early adolescence is an important risk factor of future persistent use (Vega, Zimmerman, Warheit, Apospori, \& Gil, 1993) and that early use represents a "gateway" to subsequent patterns of problem AOD use. The prevalence of use, however, differs significantly among ethnic and racial groups. Among sixth and seventh grade boys, Cubans and other Hispanics had a higher tendency to use illicit drugs, while African Americans and nonHispanic Whites reported higher levels of alcohol, tobacco, and inhalant use (Vega et al., 1993). Hispanic adolescents are an appropriate group for examining the culture-drug use relationship because they are from an ethnic group that has strong prohibitions against illicit drug use and they are highly familistic (Vega, Gil, \& Wagner, 1998). 
Studies have been conducted to measure whether acculturation levels are predictive of AOD use and there is some data on differences in Hispanic children's propensity and patterns of AOD use across differing levels of acculturation. For example, Vega, Gil, and Zimmerman (1993) found that while foreign born CubanAmerican boys had significantly lower levels of lifetime alcohol and cigarette use compared to U.S. born Cuban-American boys, they had higher levels of first time use of alcohol. Thus, when measuring acculturation as a variable related to first time AOD use, there was a significant group differences in levels of alcohol use. Specifically, monolingual Spanish speakers had lower lifetime prevalence rates than did bilingual speakers or monolingual English speakers. Vega, Gil, and Zimmerman concluded that lifetime alcohol and cigarette use increases as acculturation increases for boys of Cuban heritage in comparison to African American and White non-Hispanic boys.

\section{Summary}

In summary, one can draw from previous research that there is currently ambiguity as to which models (i.e., social influence or social and ecological model) of acculturation most accurately address acculturative stress, psychological adjustment, and substance use. Previous research on levels of acculturation and substance use has been mainly conducted with Hispanic adults, and recently adolescents have been included in this research agenda. Substance use during adolescence is a significant public health concern because early experimentation with substance use incrementally increases the likelihood of subsequent patterns of persistent use. While acculturation is gaining more prominence within ongoing research agendas, there are, at present, no measures of acculturative stressors in childhood and adolescence (Gil, Vega, \& Dimas, 1994). 
Studies seem to indicate that levels of acculturation are a key influence upon the onset or maintenance of substance use in adolescence. While Hispanic culture plays a significant protective role in AOD use, it has been found that otherwise protective traditions, norms, and values dissipate in subsequent generations as adolescents become more integrated into American culture. High levels of acculturation are associated with higher levels of AOD use, and in particular, the use of illicit drugs. Therefore, acculturation may be an important influence upon substance use among Hispanic youth. Only recently, however, has research examined direct associations between acculturation and adolescent substance use. This study begins to address this lack of research by specifically examining levels of acculturation and substance use, in addition to using acculturation variables as factors in analyses of positive change resulting from a therapeutic intervention. Hispanic male adolescents will be the focus of these analyses as males acculturate faster than females. In the next section of the literature review, past research will be reviewed, including pertinent empirical studies to support the design, implementation, analysis, and significance of the proposed research study. Review of Current Research on Acculturation and Therapeutic Interventions. Social and Linguistic Acculturation. Participants' language preferences are usually assessed in specific measures of acculturation status. Among Hispanics, language use may shift completely to English, become bilingual, or remain predominantly Spanish. Adolescents who report speaking only Spanish to friends, family, as well as in school, will be much more likely than those speaking a greater proportion of English to adhere to family practices and values that are consistent with Hispanic culture. Therefore, lower drug use among less acculturated Hispanic/Latino 
adolescents has been attributed to a protective quality of their culture of origin and their immersion in social or family systems that provide social control antithetical to problem behaviors, including substance use (Vega, Gil, \& Wagner, 1998).

Epstein et al. (2001) found that adolescents who spoke English with their parents reported greater marijuana use compared to adolescents who spoke mainly Spanish with their parents, and boys reported more frequent marijuana use than girls, alcohol patterns were not investigated in this study as one of the categories of polysubstance use. Their study also found that linguistic acculturation significantly affected patterns of polysubstance use. These findings suggest that not only are adolescents becoming more socially adjusted to the dominant culture, they are also becoming more linguistically acculturated and are losing contact with their strictly Hispanic culture. Linguistic acculturation and separation from speaking Spanish with parents or other family members can be an additional cause of acculturation stress within the family unit. High levels of stress in families may cause adolescents to separate themselves from their family and depend increasingly on peers, who may be potent social influences for engaging in delinquent behavior.

Risk and Predictive Factors. Specific risk factors have been identified as predictors of future AOD use. However, some of these factors are unique, in that they do not generalize across ethnic or racial groups. Bry et al. (1982) stated that drug use is best understood as a general coping mechanism, and as a result, the quantity of risk factors, rather than any unique combination predisposes adolescents to drug use. However, it is important to state that unique personal characteristics of individuals and their 
environments vary widely and alter the probability of maladaptive developmental outcomes.

Differences in ethnic and racial groups and the risk factors they are exposed to will significantly increase or decrease the probability of future substance use. When comparing sixth and seventh grade boys who were African Americans, non-Hispanic Whites, Cubans and other Hispanics, Vega et al. (1993) found significant risk factor differences among these different ethnic groups and that multiple risk factors influence and maintain adolescent drug use. Cubans and other Hispanics reported high levels of risk factors such as, parental smoking, perceptions of high peer substance use, and high levels of willingness to engage in delinquent behavior. While African American and non-Hispanic White adolescents scored high in similar risk factors, African Americans were more likely to actually participate in delinquent behavior. While the Vega et al. study found that the distribution of risk factors was similar across groups, Hispanics identified to have at least nine different risk factors for engaging in peer substance use, and their high levels of willingness to engage in delinquent behavior. Vega et al. also found that $35 \%$ of the Cuban boys were foreign born and resided within ethnically and culturally similar neighborhoods in Miami, and that $60 \%$ of other Hispanic boys were also foreign born and recent immigrants to Miami. Both Hispanic groups were likely to have similarly high levels of family pride in comparison to non-Hispanic Whites and African Americans.

Many researchers now consider acculturation and it stressors as key risk factors for AOD use among Hispanic/Latino adolescents. Elder at al. (2000) were particularly interested in cigarette and alcohol susceptibility and use among migrant Hispanic 
adolescents. In their sample of 660 Hispanic adolescents, between the ages of 11 and 16 , the variable of acculturation, among others, was investigated as a predictor of cigarette and alcohol use. Their study documented that $75 \%$ of the sample was first generation Hispanic and $79 \%$ preferred to speak and to complete assessment measures in Spanish. Their study found that acculturation factors were not related to alcohol susceptibility or use, but did find that Hispanic males and females have the highest rates of susceptibility of AOD use for their ethnic and gender age groups. They also found that adolescents with friends who drank alcohol, fewer self standards for drinking and reported less satisfaction with support reported greater susceptibility to alcohol. The researchers stress that although acculturation was not a predictor of AOD use in this study, it is however a risk factor since it can influence parent-child relationships and family environments.

There are various social factors and elements to which adolescents acculturate to, such as, defining themselves as an ethnic group and stigma that may now be associated to minority groups in the U.S. De la Rosa et al. (2000) states that adolescents are in constant motion in adjusting to traditional American mainstream values, while dealing with the stress of such adjustment or potential failure to adjust. For example, adolescents who have to effectively shift back and forth between different cultural orientations on a daily basis; living with racial/ethnic discrimination or lack of respect for Hispanic values and traditions; living low income neighborhoods with high levels of crime, violence, and poverty; or individual and family behaviors and support; might aid or act as a stressor in future substance use or abuse.

The Fraser et al. (1998) study on acculturation and adolescent risk behavior investigated risk factors for substance use and increased exposure to HIV and other 
STDs. Highly acculturated Hispanic female adolescents were more likely to have alcohol and drug use disorders, intravenous drug use, to use alcohol before having sex, and to use condoms with a secondary partner in contrast to less acculturated females. The results of this study among Hispanic adolescent females found that biculturalism predicted riskier sexual behavior, as well as significant relations between substance use and high risk sexual behavior in both women and adolescent girls, thus, the level of acculturation was not only a risk factor in substance use but with higher exposure to HIV and others STDs. Last, Hovey and King's (1996) study of 40 female and 30 male Hispanic adolescents found that acculturating Hispanic adolescents experience high levels of acculturative stress that puts them at higher risk for experiencing clinical levels of depressive symptoms and suicidal ideation. Results from this study demonstrate the clinical importance and implications for working with acculturating adolescents who may be experiencing high levels of acculturative stress. Markides (1981) states that the increasing patterns of suicidal behavior among Hispanic adolescents may indicate that younger Hispanics are less integrated into the family than are older Hispanic adolescents and that family emotional closeness is more important than physical closeness for protection against the development of significant mental health problems and subsequent substance use.

In summary, one can draw from previous research that the varying levels of language within the family system can be a source of acculturative stress which can lead to substance use as adolescents are becoming more acculturated to the dominant culture and are losing some of their Hispanic values and traditions. Studies also showed that varying levels of acculturation and risk and predictive factors either facilitate or impede 
adolescents' subsequent substance use. Studies showed that higher levels of acculturative stress lead to higher levels of depressive symptoms, suicidal ideation, risky sexual behavior, and willingness to engage in deviant behaviors. These finding suggest the need for research on clinical interventions designed for working with this population that provide guidance on how to best serve them and their needs.

\section{Guided Self Change.}

Substance abuse interventions are critical for interrupting the onset and continued use of illicit substances, and for providing youth with skills necessary to increase their educational and social development. Vega, Gil, and Zimmerman (1993) suggest that interventions for alcohol and cigarette use can start as early as the third or fourth grades. This consideration is developmentally appropriate considering the amount of exposure children and adolescents have to substance use. The concept of brief motivational interventions, such as Guided Self Change, is discussed by Tubman, Wagner, Gil, and Pate (2002), as an appropriate strategy for working with delinquent substance abusing adolescents. Guided Self Change (GSC) uses behavioral change principles, motivational engagement strategies, and develops substance abuse reduction goals based on each client's personal experiences and needs. Therefore, this technique can be tailored on an individual basis and it has the capability to work with a wide range of adolescent populations.

This treatment modality also helps clients to recognize factors associated with substance use and what barriers to, and supports for their recovery are present. This technique is highly dependent on behavioral techniques, which have been shown to be more effective to reduce problem behaviors, among adolescents with conduct problems 
(e.g., Mulvey, Arthur, \& Reppucci, 1993). Another major influence on the development of GSC treatment was the finding that one session of advice and counseling was more effective in addressing alcohol problems of mild and moderate severity than more traditional and intensive treatments. In addition, the importance of client's motivation to change in determining treatment outcome, and the social learning premise that allowing goal choice increases an individual's commitment to a goal (Sobell \& Sobell, 1995).

Tubman, Wagner, Gil, and Pate (2002) describe the GSC interventions that are used in this study, which are divided into five sessions. Session 1 involves the rationale for GSC treatment, providing personalized feedback on current substance abuse patterns, goal setting, and completion of self monitoring exercises. Session 2 involves clients review of past week substance use, examining trigger situations and subsequent consequences, and discussion of perceptions of problem of substance use. In session 3 past week use is again reviewed as well as examination of feelings and experiences that influence problem behaviors, social skills training, developing action plans for dealing with trigger situations, and the development of a change plan. Session 4 includes review of past week use, second assessment of situational confidence profile, and general discussion of causes of stress, coping and prevention of stress. In the final session, goals of change are reviewed, as well as past week use, developing short- and long-term goals, re-evaluation of client's perception of substance use problem, and social supports to maintain change. The GSC model can also be easily modified to accommodate family member into the intervention process by incorporating family members as a source of support through out the therapeutic intervention. It has been asserted that regarding less acculturated Hispanic adolescents, integrating family support into interventions may be 
extremely beneficial, since the traditional values and familialism are still intact (De La

Rosa et al., 2000; Gil, Vega, \& Dimas, 1994; Vega, Gil, \& Zimmerman, 1993).

Hispanic/Latino populations tend to be highly familistic. Integrating the family unit into selective interventions may prove to be extremely beneficial for all involved. It has been found that among at-risk inner city youth, adolescents and families that received familybased prevention counseling showed immediate improvement, specifically with increases in self-concept, school bonding, and family cohesion and decreases in adolescents' reports of antisocial behavior by close friends (Hogue, Liddle, Becker, \& JohnsonLeckrone, 2002).

\section{Relapse Prevention and Brief Situational Confidence}

One key component of effective substance abuse intervention programs is that of relapse prevention. Relapse prevention employs cognitive restructuring strategies to assist clients in modifying their attributions for, and preparation for the relapse process (Larimer, Palmer, \& Marlatt, 1999). Cognitive restructuring helps clients to view lapses not as failures on their part but as a signal that increased planning and more effective coping skills in similar situations are necessary. In addition, this strategy is useful for removing any further self-blame and negative emotions that may take a lapse to a full blown relapse. It is important to note the difference between a lapse and a relapse. Larimer et al. (1999) state that a lapse is the first violation of the abstinence goal, while a relapse is a return to former pattern of uncontrolled drinking/using or the abandonment of the abstinence goal.

Those who attribute a lapse to their own personal failure are likely to experience guilt and negative emotions, which can in turn lead to further drinking or substance use 
episodes. In contrast, those who attribute a lapse to situations out of their control (i.e., "I have no will power and will never be able to stop") are also more likely to experience relapse, compared to those who attribute the lapse to their inability to effectively cope with high risk situations. Larimer et al. (1999) give examples of potentially high risk situations that can encourage a lapse or relapse such as: negative emotional states, situations that involve another person or a group of people, social pressures, and positive emotional states (e.g., celebrations).

The conceptual model supporting relapse prevention treatment is based on the categorization of "triggers" emotions, i.e., events, and situations that can lead to a lapse or relapse. This notion provided the impetus to create the Brief Situational Confidence Scale (BSCQ). Assessing clients situational confidence when faced in possible relapse situations is an effective mediator in treatment efficacy, therapist and client can work together on the possibility of a relapse in sessions and therapists can "coach" clients to more effective ways of handling their confidence.

The authors' stated limitations of their study included the potential lack of generalizabilty to more severely dependent, court mandated, or adolescent populations. The generalizabilty of the measure must still be determined. In addition, the degree to which BSCQ scores predict relapse or post treatment levels of use is unknown for adolescent treatment populations. Kirisci and Moss (1997) applied the 39 item SCQ with the 8 subscales to adolescents and found that the eight factor model fit the data in the same pattern as was found in adults. They recommend its use as an effective assessment tool when working with at-risk adolescent populations. The brief version of the SCQ was also administered to a prison sample in a study done by Barber, Cooper, and Heather 
(1991). They found that it was an effective tool for assessing high-risk situations for inmates, as well as a useful evaluation tool for determining the effectiveness of treatment for AOD use. Kirsci et al. (1996) found that when sampling 662 adolescents between the ages of 12 and 18 years old, with the 39-item SCQ version normally administered to adults, each section of the SCQ measured what it intended to measure and all items of the SCQ were highly discriminating. Implying that there is no need to revise the 39 item SCQ for adolescents. Kirsci et al. also found that the SCQ is accurate in assessing selfefficacy among alcohol-abusing and deviant adolescent populations.

Clinically, it is important to measure self-efficacy as part of a prevention or treatment strategy for AOD abuse. The BSCQ can be an effective tool for helping to create an effective relapse prevention plan since it presents the individual with trigger situations that may lead them to a relapse. It thus, is hypothesized that in a sample of Hispanic adolescents being treated for substance use, the BSCQ will show the same patterns of change across time (as seen with previous research), with one key component affecting change: level of acculturation. In the therapeutic setting, for those individuals receiving familial treatment, it is expected that adolescents who are less acculturated and have stronger ties to traditional Hispanic beliefs will be likely to report greater situational confidence for resisting an AOD relapse when the BSCQ is administered a second time after 3 sessions of treatment.

Thus, the proposed study will attempt to use the limitations of existing literatures regarding the use of the BSCQ on a more dependent, court mandated adolescent population, as was mentioned as a limitation in the Breslin et al. study. Participants' self efficacy for resisting relapse will be assessed when adolescents are administered a second 
time, during therapeutic intervention after the third counseling session. It is expected that the varying levels of acculturation will factor as a component of change over time in BSCQ scores. Further, it is expected that for those Hispanic adolescents who are receiving family treatment it is expected that change across time on BSCQ scores will be likewise positive.

\section{The Proposed Study: Research Aims and Hypothesis}

Based on this review of the literature, the following two aims have been identified for investigation in this study.

Aim I: To investigate if level of acculturation significantly moderates treatment impact on changes in situational confidence scores. Based on previous studies, it is hypothesized that levels of acculturation will moderate the impact of GSC treatment on BSCQ scores. Specifically, low levels of acculturation will moderate for positive changes in situational confidence scores and high levels of acculturation will moderate negative or change in situational confidence scores.

Aim II: Investigate the degree to which nativity moderates the impact of GSC on BSCQ scores, within the family-involved guided self change treatment condition. Based on current literature, Hispanic males in the family-involved treatment condition who are born outside of the United States and who are less acculturated to the dominant culture are expected to have a positive change in BSCQ scores than U.S. born, high acculturated adolescents. 
Figure I

Acculturative Model of Immigrant and US Born Hispanic Adolescent Drug Use (Vega \& Gil, 1999.)
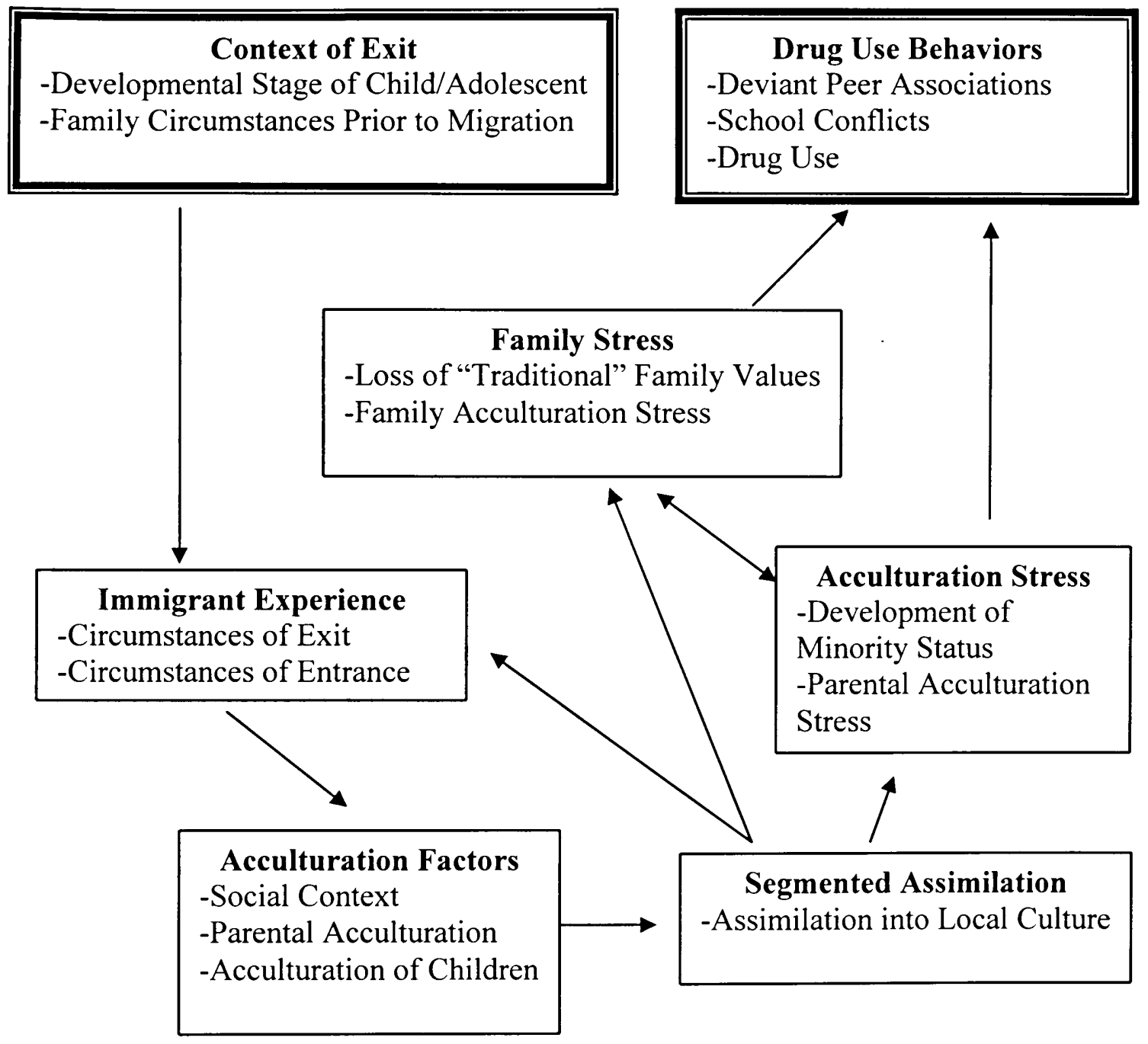


\section{CHAPTER III}

\section{METHODOLOGY}

\section{Participants}

The longitudinal design of the ATTAIN program includes a randomized clinical trial (RCT) to investigate the efficacy of a brief motivational, behavioral manualized treatment that is hypothesized to be efficacious for use with minority juvenile offenders with alcohol and/or substance abuse (e.g., marijuana abuse) problems. The age range selected for this study is based on the age inclusion criteria of the ongoing project, i.e., 14 to 18 years of age. This study will focus solely on Hispanic/Latino males for because there are insufficient numbers of female offenders in the ongoing treatment program to allow for appropriate statistical analyses.

The full sample included 242 adolescent Hispanic males participating in an ongoing research study being conducted at Florida International University, "Alcohol Treatment Targeting Adolescents In Need (ATTAIN)". However, the following factors led to a reduction in sample size; participants not starting treatment, participants dropping out of treatment, participants referred to more intensive substance abuse programs. Lastly, at the inception of program 26 participants received different versions of BSCQ and were not included in this evaluation.

During course of program the BSCQ measure that was administered was changed from an 8 -item response set to a 6 -item response set. The rationale for changing the BSCQ was that adolescents were not responding well to two items on the BSCQ. The questions that were removed tapped into the adolescent's confidence about their ability to resist the temptation and urge to drink or use ("I remembered how good it tasted", "I 
found a bottle of my favorite alcohol", etc.) and their confidence when they wanted to test their own control over their will to drink alcohol or use drugs ("I convinced myself I was a new person and could take a few drinks", "I wondered about my self control over alcohol and felt like having a drink to try it out", etc.).

In addition, participants needed to meet several criteria to be considered for inclusion in this research study. First, they had to endorse Hispanic/Latino identity on demographic questionnaire. Second, they had to be males between the ages of 14 and 18 years old. This age range was chosen for three reasons. First, the students in this age range are more likely to be experiencing the same developmental transitions. Second, they are in the critical period just prior to the transition to early adulthood. Third, participants needed to have completed at least the therapeutic component of program and completed the Brief Situational Confidence Questionnaire at Time 2 (completed in session 4 out of a possible 5 sessions). Final sample size of participants was 174 between the ages of $13-19,(m=16)$.

Descriptive analysis was used to summarize information about the sample's characteristics using items from the demographic information measure (age, place of birth, year of arrival to US, socio-economic status, etc.). Sample characteristics are summarized in Table 1.

The ethnic composition of the males in the sample varied; $69.5 \%$ of participants were born in the United States, and 31.5\% were born abroad, mostly in Hispanic/South American countries. Those who were born abroad arrived to the United States between 1984 and $2002(m=1993)$. Regarding ethnic identity, 33.3\% of participants identified themselves as Hispanic and $20.1 \%$ identified as Cuban-American as the next largest sub- 
sample, followed by $6.9 \%$ identifying themselves as Hispanic-American, and the remaining $39.7 \%$ identifying themselves as various other Hispanic groups (i.e.,

Colombian, Cuban, Nicaraguan, Mexican, etc.). Approximately $85 \%$ of participant's mothers and $90 \%$ of fathers were born outside of the United States. Approximately $88 \%$ of participants live at home with at least their mother while the remaining $12 \%$ reported living with father, other relatives (aunt, uncle, grandparents), or non-relatives. Lastly, $22.4 \%$ of participants were court mandated to participate in ATTAIN program.

Table 1

Hispanic Participants Background Information

\begin{tabular}{l} 
Variable \\
\cline { 2 - 3 }
\end{tabular}

Ethnicity

Hispanic White

$161 \quad 92.5 \%$

Hispanic Black

$9 \quad 5.2 \%$

Other

$4 \quad 2.3 \%$

Age

13 yrs.

$2 \quad 1.1 \%$

14 yrs.

$14 \quad 8.0 \%$

$15 \mathrm{yrs}$.

$25 \quad 14.4 \%$

$16 \mathrm{yrs}$.

$45 \quad 25.9 \%$

$17 \mathrm{yrs}$.

$71 \quad 40.8 \%$

18 yrs.

$15 \quad 8.6 \%$

$19 \mathrm{yrs}$.

$2 \quad 1.1 \%$

Born in the United States

Yes

121

$69.5 \%$

No

$53 \quad 30.5 \%$

Mother Born in the United States

Yes

$26 \quad 14.9 \%$

No

74

$85.1 \%$

Father Born in the United States

Yes

$17 \quad 9.8 \%$

No

$83 \quad 90.2 \%$

Participant Program Condition

Individual

$54 \quad 31.0 \%$

Family

52

$29.9 \%$

Choice

43

$24.7 \%$

Waitlisted

25

$14.4 \%$

Participant Program Treatment

Individual

Family

$117 \quad 67.2 \%$

$57 \quad 32.8 \%$ 


\section{Procedures}

Participants of this program were recruited and referred from juvenile delinquency probation or diversion programs, affiliated with the Department of Health Services and Department of Juvenile Justice in Miami-Dade County. Participants placed on probation or in diversion for offenses such as possession of marijuana, school truancy, trespassing, and school conflicts who reported substance use or abuse were referred to the ATTAIN program as part of sanctions for the charges placed against them. Several participants were also referred from school agencies in the county, or they joined the program as self-referrals who heard about ATTAIN from word-of-mouth advertisement of the program. To ease levels of attrition, participants were remunerated for their participation with gift certificates of increasing increments at follow-up assessments and with a certificate of completion at the post therapy assessment. The measures used in this study were taken from the survey currently being used for assessments in the ongoing project. To participate in the project, students must have obtained parental consent as well as given consent themselves. All three measures; Demographic Information, Brief Situational Confidence Questionnaire (BSCQ), and Hispanic/Latino acculturation; were administered to all participants at Time 1 (pre-intervention). At Time 2 (postintervention, after completing three therapeutic interventions) the BSCQ is administered again to measure situational confidence for resisting an AOD relapse.

\section{Measures}

Demographic Information. The demographic information questionnaire is a 29item questionnaire used to gather information about participants including their age, 
gender, grade in school, family income, ethnic identification, parents' and participants' country of origin, and participant and family activities.

Brief Situational Confidence Questionnaire (BSCQ). The BSCQ is a modified version of the original 100-item Situational Confidence Questionnaire (SCQ; Annis, 1986) which was designed to tap into eight self-efficacy situations clients report as being precipitants to relapse. However, because the original SCQ is lengthy and its scoring and graphing systems are quite complicated, an 8-item brief SCQ (BSCQ) was created. The eight items are derived from Marlatt's relapse prevention model (Marlatt \& Gordon, 1985) and correspond to the subscales defined by Annis and Davis (1988) for the SCQ100. These subscales are: (1) negative emotional states (e.g., "If I am afraid that things won't work out"); (2) negative physical states (e.g., "If I have trouble sleeping"); (3) positive emotional states (e.g., "If everything were going well"); (4) interpersonal conflict (e.g., "If I had an argument with a friend"); (5) social problems (e.g., "If I have problems at school or work"); and (6) positive social states (e.g., "If I wanted to celebrate with a friend"). Responses are provided on 10-point Likert scales (i.e., 10\% increments, from $0 \%=$ not at all confident to $100 \%=$ completely confident). The Cronbach's alpha coefficient was 0.85 for the BSCQ indicating a high level of internal consistency for this 8-item instrument (Breslin, Sobell, Sobell \& Agrawal, 2000).

Hispanic/Latino Acculturation. This is a 16 item measure of acculturation based on the research of Vega and Gil (1998), Felix-Ortiz et al. (1994), Cueliar et al. (1980; 1995), and Szapocznik at al. (1978). This measure identifies individuals as either bicultural or monocultural in terms of their ethnic identity, such as recognizing themselves as solely Hispanic/Latino or American-Hispanic/Latino. This measure was 
pilot tested with focus groups and appears to have high internal consistency with a Cronbach's alpha coefficient of 0.86 . It also measures ethnic identification and language use (English vs. Spanish) in multiple contexts to compute adolescents' varying levels of acculturation (high and low acculturated or bi-cultural). 


\section{CHAPTER IV}

\section{RESULTS}

The results in this chapter summarize information about Hispanic males surveyed as part of the Alcohol Treatment Targeting Adolescents In Need (ATTAIN) project ( $n=$ 174). The ranges, means, and standard deviations of the dependent variables are shown in Table 2. For all tested hypotheses, a probabilistic level of confidence was set at $p<.05$. Research aims, hypotheses, and results are summarized in Table 5.

Frequency of Change in BSCQ Scores

It was expected that during the course of treatment, BSCQ scores would change from pre- to post-interventions for the participants. The utilization of the Guided Self Change approach was a strategy for the adolescents themselves to work through change and make positive reductions in their substance use. When presented with hypothesized relapse situations, summarized in the BSCQ, adolescents are hypothesized to be more capable to assess their capacity to change when administered the BSCQ a second time, after treatment. As shown in Table 2, adolescents reported significant changes in BSCQ scores in a positive direction following treatment across all possible relapse situations.

When asked if adolescents would use alcohol or drugs while having an unpleasant emotion, $8 \%$ reported they would definitely use at Time 1 (pre-intervention), while at Time 2 (post-intervention) the percentage dropped to $0.6 \%$. Regarding use at a time of physical discomfort, $6.9 \%$ of adolescents reported they definitely would use at Time 1 , and at Time 2 , only $1.1 \%$ would definitely use. During times of pleasant emotions, $16.1 \%$ report they would definitely use alcohol or drugs at Time 1 , while $3.4 \%$ reported they would definitely use at Time 2 . In times of conflict with others, $5.2 \%$ reported they 
definitely would use when assessed at Time 1 , while $1.1 \%$ definitely would use at Time 2. When presented with social problems at school or at work, $6.9 \%$ of adolescents assessed reported they definitely would use alcohol or drugs at Time 1, and when assessed again at Time 2, $0.6 \%$ reported that they definitely would use. Last, the highest degree of change in scores was found the relapse situation when having pleasant times with others. At Time 1,27.6\% of adolescents reported that they definitely would use alcohol or drugs when having pleasant times with others. However, at Time 2 that proportion decreased to $4.6 \%$ of adolescents reporting they would definitely use alcohol or drugs. A general linear model within subjects analysis of variance (ANOVA) design was run and found that the overall BSCQ change was significant $F(1,173)=56.74, p<$ .001. Table 2 demonstrates the significance change for each BSCQ item from Time 1 to Time 2.

Table 2

Descriptive Statistics for BSCQ variables

\begin{tabular}{|c|c|c|c|c|c|}
\hline Variable & $n$ & Mean & $\begin{array}{c}\text { Standard } \\
\text { Deviation }\end{array}$ & $F$ & $P$ \\
\hline Time 1: Unpleasant Emotion & 174 & 6.65 & 3.341 & 24.24 & 0.001 \\
\hline Time 2: Unpleasant Emotion & 174 & 8.03 & 2.187 & & \\
\hline Time 1: Physical Discomfort & 174 & 8.07 & 3.177 & 3.312 & 0.070 \\
\hline Time 2: Physical Discomfort & 174 & 8.60 & 2.407 & & \\
\hline Time 1: Pleasant Emotions & 174 & 5.90 & 3.783 & 43.83 & 0.001 \\
\hline Time 2: Pleasant Emotions & 174 & 8.21 & 2.697 & & \\
\hline Time 1: Conflict with Others & 174 & 7.27 & 3.213 & 16.85 & 0.001 \\
\hline Time 2: Conflict with Others & 174 & 8.81 & 5.168 & & \\
\hline Time 1: Social Problem at School or Work & 174 & 7.52 & 3.345 & 12.05 & 0.001 \\
\hline Time 2: Social Problem at School or Work & 174 & 8.49 & 2.117 & & \\
\hline Time 1: Pleasant Times with Others & 174 & 3.84 & 3.339 & 92.85 & 0.001 \\
\hline Time 2: Pleasant Times with Others & 174 & 7.00 & 3.176 & & \\
\hline
\end{tabular}




\section{Levels of Acculturation}

As previously mentioned, participants' language preferences are usually assessed in measures of acculturation status. Among Hispanics, language use may shift completely to use of English, they may become bilingual, or they may remain predominantly Spanish speaking. The Hispanic Ethnic Identity measures of ethnic pride, group identification, acculturative stress, and language preference were used as proxy variables for acculturation. Separate frequency and factor analyses were run for the ethnic pride, group identification, acculturative stress and language preference subscales of Hispanic Ethnic Identity measure. The subscale of the measure identifying levels of acculturation based on ethnic pride had a Cronbach alpha of .71. Scores for level of group identification had an alpha $=.75$, acculturation based on acculturative stress had an alpha $=.70$, and acculturation measured by language preference had an alpha $=.68$.

Participants were identified as highly acculturated, less acculturated or bi-cultural based on responses on Hispanic Ethnic Identity measure. Table 3 shows participants' acculturation levels based on ethnic pride, group identification, acculturative stress, and language preference.

Questions on the Hispanic Ethnic Identity measure ranged from 1 (strongly agree) to 7 (strongly disagree). Ethnic pride and group identification responses ranging between 1 and 3 were considered to be in the low acculturated range, between 3 and 5 in the bicultural range, and between 5 and 7 in the highly acculturated range. Acculturative stress was measured on a scale between 1 and 4, participants responding between 1 and 2 reported low acculturative stress while participants responding between 2 and 4 reported high acculturative stress. For the language preference questions, the last two questions 
were not included in final factor analysis (i.e., what language are the magazines you read?, and what language are the movies, $\mathrm{TV}$, and radio programs you like to watch and listen to the most?) due to it lowering the reliability of the overall language preference measure.

Participants identified themselves as either being highly or less acculturated depending on their level of ethnic pride. Participants surveyed who reported low levels of acculturation, regarding ethnic pride, accounted for $79.3 \%$ of the sample, $17.8 \%$ identified themselves as bicultural, while the remaining $2.9 \%$ were identified as being highly acculturated. These figures suggest that a majority of the participants still have high levels of ethnic pride towards their identified ethnic group, and are therefore, represented as less acculturated Hispanic adolescents.

Participants identified themselves as either having high or low levels of acculturation depending on their level of group identification. Participants surveyed who reported their low levels of acculturation regarding group identification, accounted for $39.7 \%$ of sample and the remaining $17.8 \%$ were identified as being highly acculturated. However, the majority of the participants (42.5\%) identified themselves as bicultural with regard to their group identification. These figures suggest that a majority of the participants have been able to adapt to United States customs, traditions, and values, but yet maintain a level of their own Hispanic group identification.

Participants also identified themselves as either having high or low levels of acculturation, depending on their level of acculturative stress experienced. A majority of individuals (82.2\%) experienced little or no acculturative stress, while $17.8 \%$ of Hispanic adolescent males experienced moderate or high levels of acculturative stress. The final 
measure of acculturation was based on participants' language preference. A majority of the sample, $50.6 \%$, reported themselves to be bicultural, signifying that they use Spanish and English almost equally as their preferred language. The highly acculturated participants, those who spoke English most or all of the time, comprised $35.1 \%$ of the participants. The remaining $14.4 \%$ of participants are considered less acculturated, as they speak Spanish most or all of the time.

Table 3

Participants Level of Acculturation

\begin{tabular}{lcccc}
\hline \multicolumn{1}{c}{ Acculturation Variable } & $\mathrm{n}$ & Frequency & Mean & $\begin{array}{c}\text { Standard } \\
\text { Deviation }\end{array}$ \\
\hline & 174 & & 1.2356 & .489 \\
Acculturation based on ethnic pride & & 138 & & \\
$\quad \begin{array}{l}\text { Low Acculturated } \\
\text { Bicultural }\end{array}$ & 31 & & \\
$\quad$ Highly Acculturated & 5 & & \\
& 174 & & 1.7816 & .728 \\
Acculturation based on group identification & & 69 & & \\
$\quad$ Low Acculturated & & 74 & & \\
Bicultural & & 31 & & \\
$\quad$ Highly Acculturated & 174 & & 1.3199 & .435 \\
& & 143 & & \\
Acculturation based acculturative stress & & 31 & & \\
$\quad$ Low acculturative stress & & & \\
$\quad$ High acculturative stress & 174 & & 2.2069 & .674 \\
Acculturation based on language preference & & 25 & & \\
$\quad$ Low Acculturated & & 88 & & \\
$\quad$ Bicultural & & 61 & & \\
$\quad$ Highly Acculturated & & & & \\
\hline
\end{tabular}

BSCQ Change Due to Acculturation Variables

It was found that during the course of treatment there was a positive change in BSCQ scores from Time 1 to Time 2 in the Hispanic male population that was surveyed. Language preference, group identification, ethnic pride, and acculturative stress were the 
four variables used to determine any interactional effects on BSCQ scores over time. It was found that participant levels of group identification and language preference had a marginal impact on change in BSCQ during treatment over time. Group identification was the statistically controlled variate and a general liner model within subjects analysis of covariance design was run and found that BSCQ change was marginally significant $F(1,172)=3.455, p<.065$. Language preference was the next statistically controlled variate and a general liner model within subjects analysis of covariance design was generated and found that BSCQ change was marginally significant $F(1,172)=3.652, p<$ .058. However, group identification and language preference as an interaction over time was found to not be significant $F(1,172)=1.131, p<.289, F(1,172)=.084, p<.773$. Acculturative stress and ethnic pride did not have a significant impact on change in BSCQ scores. Acculturative stress was the third statistically controlled variate and a general liner model within subjects analysis of covariance design was completed and found that BSCQ change was not significant $F(1,172)=8.393, p<.004$. Last, ethnic pride was a statistically controlled variate and a general liner model within subjects analysis of covariance design was run and found that BSCQ change was not significant $F(1,172)=4.514, p<.035$. As it was found with group identification and language preference, acculturative stress and ethnic pride were also found to not have an interactional effect over time in BSCQ change. 
Table 4

ANCOVA for BSCQ Change across Acculturation Variables

\begin{tabular}{lccc}
\hline \hline BSCQ Change over time & Statistic & F & P-Value \\
\hline Time X Language Preference & $F$ & 0.084 & 0.773 \\
Time X Group Identification & & 1.131 & 0.289 \\
Time X Acculturative Stress & & 0.335 & 0.564 \\
Time X Ethnic Pride & & 0.003 & 0.496 \\
\hline
\end{tabular}

* For all analyses Hypothesis DF $=1.00$, Error DF, 172.00

For the last hypothesis, a two factor analysis of variance was completed to determine if there was an interactional effect between participants who were born either U.S. or foreign born and were receiving family treatment. It was found that over time both groups of participants had a change in BSCQ scores $F(1,55)=13.139, p<.001$. However, when place of birth was investigated to measure an interactional effect over time in the groups no significant change was found $F(1,55)=.030, p<.864$. Various acculturation variables had different effects over time within participant groups and there was no interactional effect for any of the acculturation variables over time. Further analysis also showed that participants' place of birth was not a factor in contributing to change in BSCQ scores among youth receiving family treatment.

Table 5

BSCQ Score Change across Nativity and Family Treatment Condition

\begin{tabular}{llccc}
\hline \hline Nativity & & Mean & SD & N \\
\hline BSCQ Pre & US Born & 38.33 & 13.71 & 43 \\
& Foreign Born & 43.36 & 15.16 & 14 \\
& Total & 39.56 & 14.11 & 57 \\
& & & & \\
BSCQ Post & US Born & 47.26 & 12.47 & 43 \\
& Foreign Born & 53.18 & 8.54 & 14 \\
& Total & 48.71 & 11.84 & 57 \\
\hline \hline
\end{tabular}


Table 6

ANOVA for BSCQ Change across Place of Birth

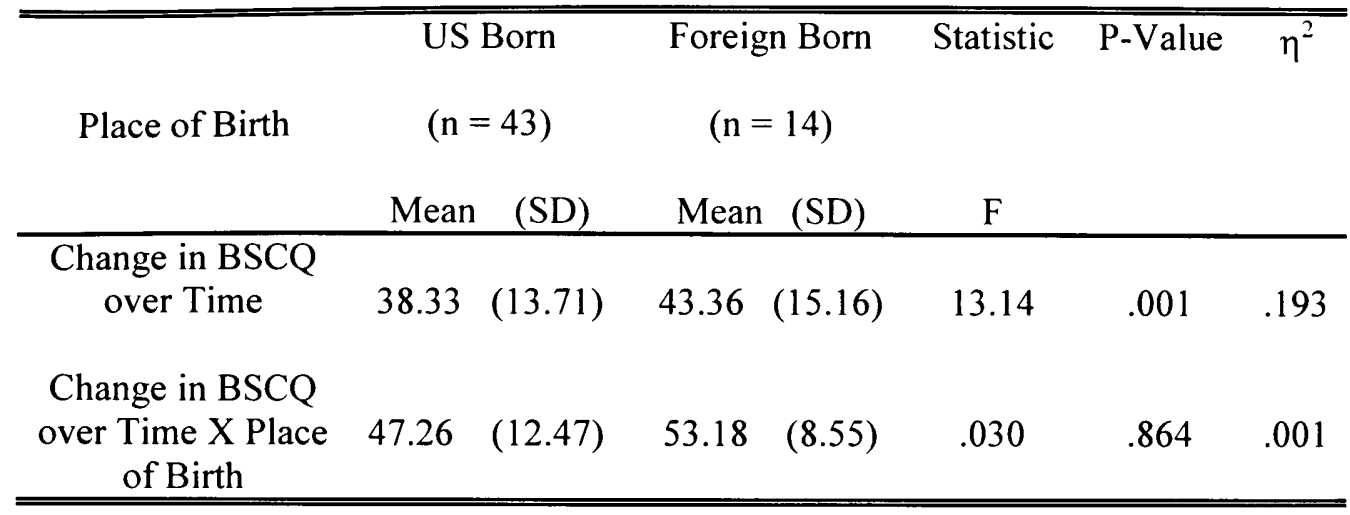

* For all analyses Hypothesis DF $=1.00$, Error DF, 55.00 


\section{CHAPTER V}

\section{DISCUSSION}

The purpose of this study was to investigate if level of acculturation among Hispanic adolescent males in delinquency probation/diversion programs impacted treatment change in a substance abuse program, specifically on the Brief Situational Confidence Questionnaire measure. This question was important to investigate because evidence suggests that there is a direct link between levels of acculturation and subsequent substance abuse. Previous research has shown that, Hispanic adolescents are at a high risk for substance use, probably due to acculturative stress that they experience after moving to a new host country, involving conflicts between the social pressures of customs and traditions of their family and the demands of new peers. Thus, it was expected that adolescents who had lower levels of acculturation would respond more positively to treatment than those who were highly acculturated or considered themselves to be bicultural.

The first hypothesis stated that levels of acculturation would moderate treatment impact as measured by situational confidence in AOD use, and that lower levels of acculturation would be predict positive change whereas higher levels of acculturation would predict no or negative change. However, it was found that adolescents changed over time in BSCQ scores regardless of acculturation level. This was true across all aspects of acculturation assessed. Adolescents with both high or low levels of acculturation in ethnic pride, group identification, acculturative stress, and language preference were found to change positively over time in their confidence to resist AOD 
use. These changes imply that various groups within the sample are changing at different times through out the course of treatment.

The second hypothesis stated that Hispanic males who were born outside of the United States and were less acculturated to the dominant U.S. culture would be more likely to have a positive change in BSCQ scores from Time 1 to Time 2 when receiving family treatment. When measuring Hispanic adolescent males who were receiving family treatment, place of birth had no statistical interactional significance in BSCQ change over time. However, both groups, U.S. and non-U.S. born adolescents, had a change over time when receiving family treatment, possibly indicating that the family condition itself was the interactional effect on change over time compared to the adolescents who did not receive the family treatment.

\section{Limitations}

There were several limitations to the current study which must be considered when interpreting the results. First, the sample size used in this study was relatively small, especially when comparing the U.S. and non-U.S. born Hispanic males, thus reducing statistical power. Analyses performed on a larger sample size of Hispanic adolescent males who received the BSCQ at both assessment times could have increased the statistical power for detecting significant change across the various acculturation variables.

Second, the validity of the results obtained from the current program may have been limited by the reliance on self-report measures. Asking participants to report their level of confidence regarding AOD use at Time 1 and then again at Time 2 may not have obtained the most accurate and truthful reports. Adolescents may feel the need to please 
the evaluator at Time 1 and the therapist at Time 2. Adolescents may also be fearful of suffering further repercussions from juvenile justice probation officers if admitting to not improving during treatment. In future studies, validity of the data obtained could be improved by obtaining reports from significant others; such as parents or other family members; peers; or other identified social support; or if length of treatment was longer than five sessions and adolescents could further explore relapse prevention exercises and triggers.

Third, the aspects of acculturation measured in this study represent some aspects of culture that may be associated with substance use, but not all. Acculturation may impact treatment, but it may have been true that the therapists administering the intervention were "culturally competent", so all youth were positively impacted.

Fourth, "acculturation" in the community context of South Florida, with high percentage of migrant population in the U.S., may not represent the context of alcohol use or treatment for other youth.

\section{Contribution to Knowledge}

Breslin, Sobell, Sobell, and Agrawal's (2000) study stated their study included the potential lack of generalizabilty to more severely dependent, court mandated, or adolescent populations. The results of this study found that measuring the degree of change in BSCQ scores from pre-intervention to post-treatment generalized the BSCQ to the court mandated and juvenile justice referred adolescents that were absent from their study. In addition, the degree to which BSCQ scores predict relapse or post treatment levels of use was unknown for adolescent treatment populations. 
The concept of acculturation is gaining more recognition in the field of substance abuse research and measures are being developed and validated to assess levels of acculturation in substance abusing populations. However, there is no current measure identifying what are acculturative stressors, or the most important risk or protective factors in children and adolescents. By improving assessments of levels of acculturation, researchers may be able to identify the important risk and protective factors for children and adolescents. In terms of key acculturation variables, adolescents participating in the program were identified as less acculturated, highly acculturated, or bicultural. Among highly acculturated adolescents, non-substance abusing peers may be more of a protective factor than the family which would be expected to be found in less acculturated or bicultural children and adolescents.

As previously mentioned, findings seem to indicate that high levels of acculturation is associated with significantly higher drug use, especially with regard to illicit drug use (Carvajal, Photiades, Evans, \& Nash, 1997; Gil, Wagner, Vega, 2000; Vega, Gil, \& Wagner, 1998). It is important to distinguish between immigrant and U.S. born adolescents' acculturation in studying the effects of acculturative stress factors on AOD use. Measuring acculturation and acculturative stress as a risk factor for drug and alcohol use has typically involved sampling and assessment of adults, and only recently has available research investigated this risk factor in the onset and maintenance of adolescent drug and alcohol problems. However, due to the small sample size, and few adolescents reporting high levels of acculturative stress within the current study, a larger study may be needed to investigate the degree to which levels of acculturative stress could impact the onset and maintenance of adolescent substance use and abuse. 


\section{Clinical Implications Based on Evidence}

When working with relapse prevention, it is helpful to work with clients to help them identify realistic situations and triggers that could lead to a relapse (Larimer, Palmer, \& Marlatt, 1999). The BSCQ measure appears to capture change related to treatment at least among the Hispanic male adolescents surveyed who participated in this study. The use of the Guided Self Change approach is one that has been shown to work well with adolescents due to its behavioral change principles, motivational engagement strategies, and the development of substance abuse reduction goals based on each client's personal experiences and needs. This technique can be tailored on an individual basis and has the capability to work with a wide range of adolescent populations (Sobell \& Sobell, 1995). As previously mentioned, the GSC model can also be easily modified to accommodate family members into the intervention process by incorporating them as a source of support throughout the therapeutic intervention as it has been asserted that with less acculturated Hispanic adolescents, integrating family support into interventions may be extremely beneficial, since the traditional values and familialism are still intact (De La Rosa et al., 2000; Gil, Vega, \& Dimas, 1994; Vega, Gil, \& Zimmerman, 1993).

The results of the study showed that when GSC is used, and the BSCQ is used to assess change, most adolescents report a significant amount of change over the course of treatment (i.e., using drug of choice everyday when assessed at pre-assessment versus not using at all at post-assessment). The use of the BSCQ measure in relapse prevention assessed participants' confidence when placed in possible trigger situations. The results of change in BSCQ score from pre-assessment to post-assessment among the participants suggest that the usefulness of the BSCQ is a potential mediator of change in treatment. 


\section{Clinical Insight}

Although this study found no conclusive evidence that change on BSCQ scores varied by levels of acculturation, in clinical settings therapists should address issues of acculturation and how it could possibly factor into substance use prevention and treatment (Larimer, Palmer, \& Marlatt, 1999). It is important for clinicians to bear in mind that stigma is placed on many Hispanic groups in the United States and therefore, clinicians should tailor therapeutic interventions based on the adolescents' levels of acculturation. Clinicians should also recognize that certain acculturative strains are specific to specific groups (Vega, Gil, \& Wagner, 1998). Factors such as having to effectively shift back and forth between different cultural orientations on a daily basis, living with racial/ethnic discrimination, lack of respect for Hispanic values and traditions, individual and family behaviors and support, might act as risk or protective factor for future substance use. For example, using the family or peer networks as protective factors as support may help adolescents through periods of change and may also significantly impact treatment outcomes (Gil, Vega, \& Dimas, 1994).

Although measures of acculturation are not always readily available, therapists should show heightened sensitivity to assess adolescents' varying levels of acculturation. For example, therapist may need extra training to be able to distinguish from normative adolescent rebellious behavior against the family and rebellious behavior due to differing speeds at which adolescents and their parents are acculturating. As stated, adolescents often acculturate at faster rates than their parents. Therefore, adolescents' rebellious behavior may be due to conflict between parents' traditional Hispanic cultures and norms and adolescents' more acculturated values. It may be that the results of this study can be 
explained by the fact that the therapist administering the GSC treatment did provide these forms of culturally sensitive treatment, so youth responded well regardless of acculturation.

In conclusion, this study was intended to investigate if varying levels of acculturation moderated treatment efficacy, which was measured by the use of the BSCQ measure. Although the hypotheses of the study were not supported, the data did find that when GSC is used with the BSCQ most adolescents reported a significant amount of change over the course of treatment. BSCQ scores went up significantly for all groups of acculturated adolescents, however, there was no moderating effect for the proxy acculturation variables that were measured. For the ATTAIN program these findings suggest that treatment is working for the Hispanic male adolescents surveyed, however, acculturation was not a mediator of change in this treatment. 
Annis, H. M., \& Davis, C. S. (1989). Relapse prevention training: a cognitive behavioral approach based on self efficacy theory. Journal of Chemical Dependency Treatment, 2, 2, 81-103.

Bandura, A. (1978). Reflections on self efficacy. In Stevens \& Smith (Ed) Substance Abuse Counseling, Theory and Practice: New Jersey: Prentice Hall.

Bandura, A., \& Walter, R. (1959). Adolescent aggression. In L. Steinberg (Ed) Adolescence: Boston: McGraw-Hill College.

Barber, J. G., \& Cooper, B. K. (1991). The situational confidence questionnaire (heroin). The International Journal of the Addictions, 26, 5, 565-575.

Breslin, C. F., Sobell, L. C., Sobell, M. B., \& Agrawal, S. (2000). A comparison of a brief and long version of the situational confidence questionnaire. Behaviour Research and Therapy, 38, 1211-1220.

Brunswick, M. (1989). More kids turning to inhalant use. In H. E. Doweiko (Ed) Concept of Chemical Dependency: California: Books/Cole.

Carvajal, S. C., Photiades, J. R., Evans, R. I., \& Nash, S. G. (1997). Relating the social influence model to the role of acculturation in substance abuse among Latino adolescents. Journal of Applied Social Psychology, 27, 18, 1617-1628.

Condiotte, M. M., \& Lichtenstein, E. (1981). Self-efficacy and relapse prevention smoking cessation programs. In Stevens \& Smith (Ed) Substance Abuse Counseling, Theory and Practice: New Jersey: Prentice Hall.

DeBellis, M.D., Clark, D. B., Beers, S. R., Soloff, P. H., Boring, A. M., Hall, J., Kersh, A., \& Keshavan, M. S. (2000). Hippocampal volume in adolescent onset alsochol use disorders. In H. E. Doweiko (Ed) Concept of Chemical Dependency: California: Books/Cole.

De La Rosa, M., Vega, R., Radisch, M. A. (2000). The role of acculturation in the substance abuse behavior of African American and Latino adolescents. Journal of Psychoactive Drugs, 32, 1, 33-42.

Elder, J. P., Campbell, N. R., Litrownik, A. J., Ayala, G. X., Slymen, D. J., ParraMedina, D., \& Lovato, C. Y. (2000). Predictors of cigarette and alcohol susceptibility and use among Hispanic migrant adolescents. Preventive Medicine: An International Journal Devoted to Practice \& Theory, 31, 2 (Pt.1), 115-123. 
Epstein, J. A., \& Botvin, G. J, \& Diaz, T. (2001). Linguistic acculturation associated with higher marijuana and polydrug use among Hispanic adolescents. Substance Use \& Misuse, 36, 4, 477-499.

Fraser, D., Piacentini, J., Van Rossem, R., Hien, D., \& Rotheram-Borus, M. (1998). Effects of acculturation and psychopathology on sexual behavior and substance use of suicidal Hispanic adolescents. Hispanic Journal of Behavioral Sciences, 20, 1, 83-97.

Gil, A. G.,Vega, W. A.,Dimas, J. M. (1994). Acculturative stress and personal adjustment among Hispanic adolescent boys. Journal of Community Psychology; 22, 1, 4354.

Gil, A. G., Wagner, E. F., \& Vega, W. A. (2000). Acculturation, familism, and alcohol use among Latino adolescent males: longitudinal relations. Journal of Community Psychology, 28, 4, 443-458.

Gullotta, T.P., Adams, G.R., \& Montemayor, R. 1994. Substance misuse in adolescents. Thousand Oaks, California: Sage Publications.

Hogue, A., Liddle, H. A., Becker, D., Johnson_Leckrone, J. (2002). Family based prevention counseling for high risk young adolescents: immediate outcomes. Journal of Community Psychology, 30, 1, 1-22.

Hovey, J. D. \& King, C. A. (1996). Acculturative stress, depression, and suicidal ideation among immigrant and second generation Latino adolescents. Journal of the American Academy of Child and Adolescent Psychiatry, 35, 9, 1183-1193.

Johnston, L. D., O'Malley, P. M., Bachman, J. G. \& Schulenberg, J. E. University of Michigan News and Information Services: Ann Arbor, MI. [On-line]. Available: www.monitoringthefuture.org; accessed 07/19/04.

Kamier, Y. 1994. Adolescent substance use: a comprehensive guide to theory and practice. New York, NY: Plenum Medical Book Inc.

Kirisci, L. \& Moss, H. B. (1997). Reliability and validity of the situational confidence questionnaire in an adolescent sample: confirmatory factor analysis and item response theory. Measurement and Evaluation in Counseling and Development, 30, 146-155.

Kirisci, L., Moss, H. B., \& Tarter, R. E. (1996). Psychometric evaluation of the situational confidence questionnaire in adolescents: fitting a graded item response model. Addictive Behaviors, 21, 3, 303-317. 
Larimer, M. E., Palmer, R. S., \& Marlatt, A. (1999). Relapse prevention: an overview of Marlatt's cognitive behavioral model. Alcohol Research and Health, 23, 2, 151 160.

Millman, R. B., \& Beeder, A. B. (1994). Cannabis. In:H. E. Doweiko (Ed) Concept of Chemical Dependency: California: Books/Cole.

Moore, S., \& Gullone, E. (1996). Predicting adolescent risk behavior using personalized cost-benefit analysis. In: L Steinberg (Ed) Adolescence. Boston: McGraw-Hill College.

Quadrel, M., Fischoff, B., \& Douglas, G. (1996). Students conceptions of learning and their use of self regulated learning strategies. In: L Steinberg (Ed) Adolescence. Boston: McGraw-Hill College.

Siepker, B.B., \& Kandaras, C.S. 1985. Group therapy with children and adolescents: a treatment manual. New York, NY: Human Sciences Press.

Steinberg, L. 1999. Adolescence. Boston, MA: McGraw-Hill College.

Tubman, J. G., Wagner, E. F., Gil, A. G., \& Pate, K. N. (2002). Brief motivational intervention for substance abusing delinquent adolescents: Guided Self Change as a social work practice innovation. Health \& Social Work, 27, 3, 208-212.

Vega, W. A., Gil, A. G., \& Zimmerman, R. S. (1993). Patterns of drug use among CubanAmerican, African-American, and White non-Hispanic boys. American Journal of Public Health, 83, 2, 257-259.

Vega, W. A., Gil, A. G., Warheit, G. J., Zimmerman, R. S., \& Apospori, E. (1993). Acculturation and delinquent behavior among Cuban American adolescents: Toward an empirical model. American Journal of Community Psychology, 21, $1,113-125$.

Vega, W. A., Gil, A. G., \& Wagner, E. (1998). Cultural adjustment and Hispanic adolescent drug use. In: WA Vega \& A Gil (Eds) Drug Use and Ethnicity in Early Adolescence. New York: Plenum Press.

Vega, W. A., \& Gil, A. G. (1999). A model for explaining drug use behavior among Hispanic adolescents. Drugs \& Society, 14, 1, 57-71.

Vega, W. A., Zimmerman, R. S., Warheit, G. J., Apospori, E., \& Gil, A. G. (1993). Risk factors for early adolescent drug use in four ethnic and racial groups. American Journal of Public Health, 83, 2, 185-190. 
APPENDIX 1 - Demographic Information Measure 


\section{DEMOGRAPHIC INFORMATION (Juvenile)}

ATTAIN

1. Indicate respondent's gender

Male $\square$ Female

2. Were you court mandated to participate in this program? $\square$ Yes $\square$ No

3. What is your birthdate?

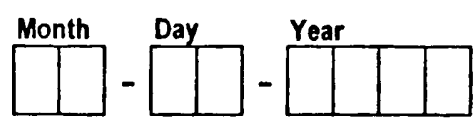

4. Where were you born?

5. In what year did you arive in the U.S.?

$\square$ U.S. $\longrightarrow$ Go To \#6 $\square$ Mexico

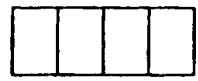

$\square$ Cuba

Dominican Republic

$\square$ Nicaragua

El Salvador

Colombia

Other

Puerto Rico

6. What is your race/ethnicity?

7. What is the highest grade you have completed?
$\square$ White non-Hispanic
$\square$ Hispanic White
Hispanic Black
African American
Other

$\square$ 6th grade $\square$ 10th grade

$\square$ 7th grade $\square$ 11th grade

$\square$ 8th grade $\square$ 12th grade

$\square$ 9th grade $\square$ Other:

8. During the past school year did you attend school?
$\square$ Yes $\longrightarrow$ Go To \#11
$\square$ No

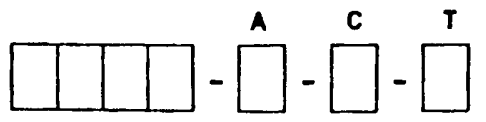


$\therefore \cdot \frac{\square 5776}{596}$

9. Which of these best describes why you stopped going to school?

To go to work

$\square$ To help support the family

$\square$ Got bored, did not get anything out of it

Saw no future in it

Did not get along with the other students

Did not get along with teachers

$\square$ I was pregnant or became a father/mother

Many of my friends had dropped out
I did not feel safe at school

Take care of ill/disabled family member

Got in trouble with the law

Had a drug or alcohol problem

Was getting poor grades/failing

Left the area

Was expelled

Other reason:

\section{INTERVIEWER CHECKPOINT}

IF ANSWERED "WAS EXPELLED"

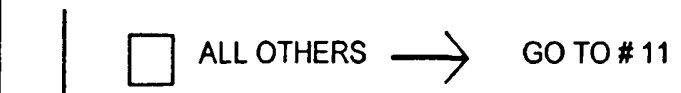

10. What was the major reason you were expelled? 11. Overall, what grades do you get most of the time?

$\square$ Fighting in school

$\square$ Selling drugs

$\square$ Using drugs

$\square$ Bringing a weapon to school

Excessive truancy (skipping)

$\square$ Other:
12. Did you ever repeat a grade?

13. Which grade did you repeat?

$\square$ Yes
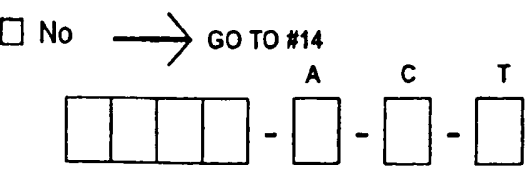
14. I currently live with (check all that apply):

$\begin{array}{ll}\square \text { My mother } & \square \text { My aunt } \\ \square \text { My father } & \square \text { My uncle } \\ \square \text { My stepmother } & \square \text { Other relatives } \\ \square \text { My stepfather } & \square \text { Non-relatives } \\ \square \text { My sister } & \square \text { Girffriend/boyfriend } \\ \square \text { My brother } & \square \text { Alone } \\ \square \text { My grandmother } & \square \text { Homeless } \\ \square \text { My grandfather } & \end{array}$

15. Of the people with whom you live, who takes care of you on a day-to-day basis?

$\begin{aligned} & \square \text { Mother } \\ & \square \text { Father } \\ & \square \text { Mother and Father }\end{aligned}$
$\square$ Grandmother
$\square$ Grandfather
$\square$ Uncle
$\square$ Aunt
$\square$ Other relative
$\square$ Non-relative guardian
$\square$ Other:

15a. For how long has this person been taking care of you?

16. Do you belong to a church, temple, or synagogue?

17. How religious are you?
$\square$ Yes
$\square$ No
Used to belong

$\square$ Very

$\square$ Moderately

A little

Not at all

18. How often do you attend services at a church/synagogue?

\begin{tabular}{|c|c|c|c|c|}
\hline Never & $\begin{array}{c}\text { Once or } \\
\text { twice a } \\
\text { year }\end{array}$ & $\begin{array}{l}\text { Once a } \\
\text { month }\end{array}$ & $\begin{array}{c}\text { Once a } \\
\text { week }\end{array}$ & $\begin{array}{c}\text { More than } \\
\text { once a } \\
\text { week }\end{array}$ \\
\hline$\square$ & $\square$ & $\square$ & $\square$ & $\square$ \\
\hline
\end{tabular}

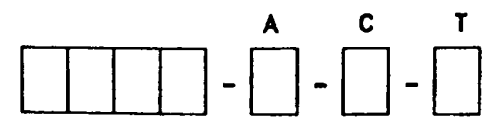


39476

19. Where was your mother born?

$\begin{array}{ll}\square \text { Cuba } & \square \text { Dominican Republic } \\ \square \text { Nicaragua } & \square \text { El Salvador } \\ \square \text { Colombia } & \square \text { Don't Know } \\ \square \text { Puerto Rico } & \square \text { Other }\end{array}$

21. How far did your father go in school?

$\square$ Graduate professional training

College graduate

$\square$ Some college

$\square$ Vocational school

$\square$ High school graduate

$\square$ Partial high school

$\square$ Junior high school graduate

$\square$ Less than 7 years of school

$\square$ Don't Know
20. Where was your father born?
$\square$ U.S.
Mexico
$\square$ Cuba
Dominican Republic
$\square$ Nicaragua
El Salvador
$\square$ Colombia
Don't Know
$\square$ Puerto Rico $\square$ Other

22. How far did your mother go in school?

$\square$ Graduate professional training

$\square$ College graduate

$\square$ Some college

$\square$ Vocational school

$\square$ High school graduate

$\square$ Partial high school

$\square$ Junior high school graduate

$\square$ Less than 7 years of school

$\square$ Don't Know

23. Does your father currently work? $\square$ Yes

$\square$ No $\longrightarrow$ GOTO 25

Don't know/No contact

24. Please specifically describe the kind of work he currently does?

25. Does your mother currently work?
$\square$ Yes
$\mathrm{D}$ No $\longrightarrow$ GO TO \#27
$\square$ Don't know/No contact

26. Please specifically describe the kind of work she currently does?
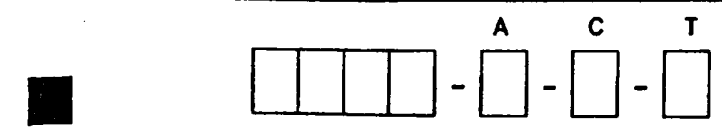
- 19

27. Do you belong to any social organization or community group? (sport team, school club, etc.).

$$
\begin{aligned}
& \square \text { Yes } \\
& \square \text { No }
\end{aligned}
$$

28. Describe the organization or group:

29. In what kinds of activities does your family routinely engage?

$\square$ Go to movies together

$\square$ Go to the park

$\square$ Watch television together

$\square$ Go to the beach

$\square$ Go on vacations

$\square$ Go out to eat

$\square$ None

Other: 
APPENDIX 2 - Brief Situational Confidence Questionnaire 


\section{Brief Situational Confidence Questionnaire (BSCQ Update)}

Imagine yourself as you are right now in each of the following types of situations. Show on the scale below how sure you are right now that you would not drink or use your primary drug in each situation by placing an " $X$ " along the line, from "Definitely Would Use" to "Definitely Would Not Use", as in the example below.

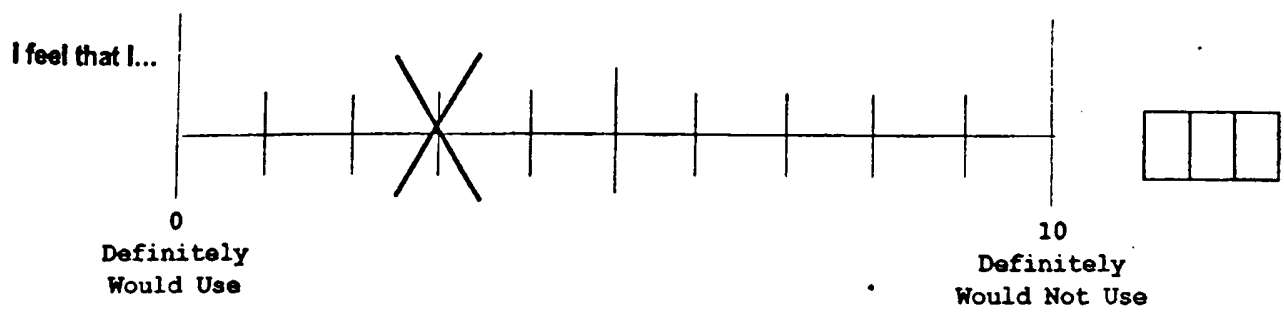

This person is pretty sure that he/she would use.

Right now I would not drink or use my primary drug in situations involving...

1. Unpleasant Emotions (e.g. if I am afraid that things won't work out, if I am angry at the way things turn out, if other people treat me unfairty).

I feel that $1 .$.
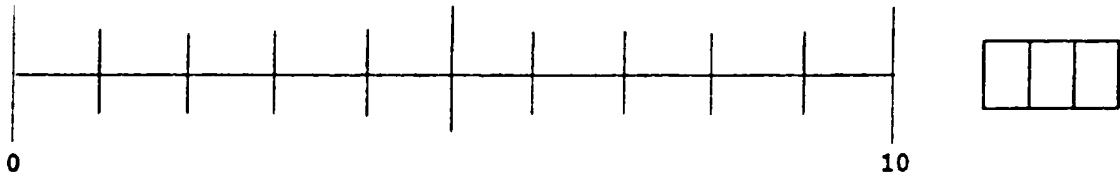

Definitely

Definitely

Would Use

Would Not Use

2. Physical Discomfort (e.g. if I have trouble sleeping, if I feel tired and want to stay alert, if my stomach feels upset or nervous).

I foel that $1 . .$.
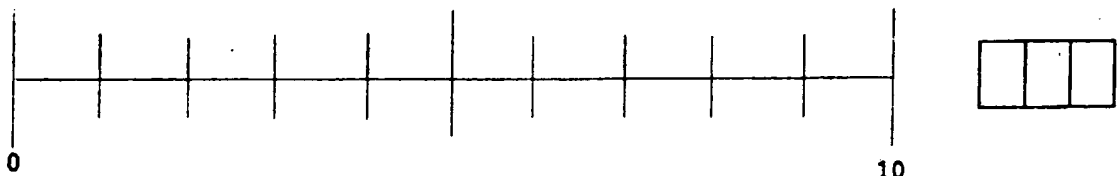

Definitely

10

Would Use

Definitely

Would Not Use

3. Pleasant Emotions (e.g. if I felt confident and relaxed, if everything is going well, if I feel satisfied with something I had done).

I feel that I...

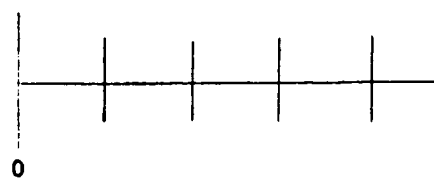

Definitely

Would Use

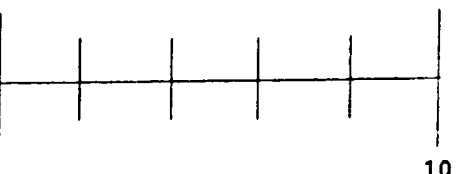

10

Definitely

Would Not Use
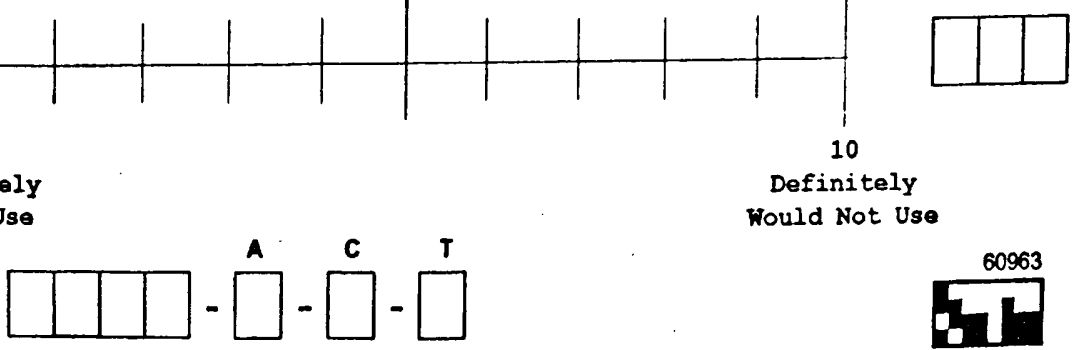

60963

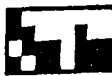


Right now I would not drink or use my primary drug in situations involving...

4. Conflict with Others (e.g. if I have an argument with a friend, if someone criticizes me, if other people around me made me tense).

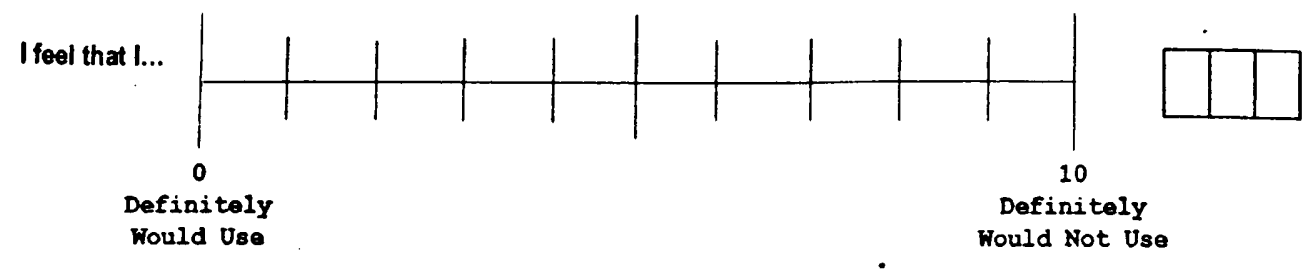

5. Social Problems At School or Work(e.g. if I have problems at school or work, if pressure builds up because of the teachersor the boss, if I am not getting along with others).

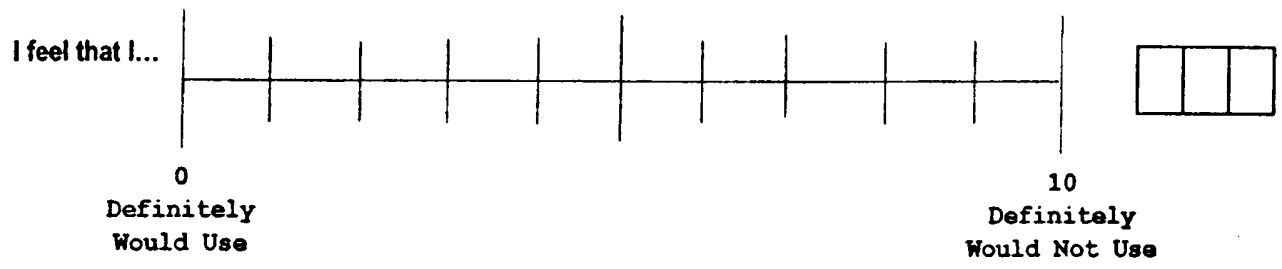

6. Pleasant Times With Others (e.g. if I am enjoying myself at a party and want to feel even better, if I want to celebrate with a friend, if I meet a friend and he/she wants to drink or use together).
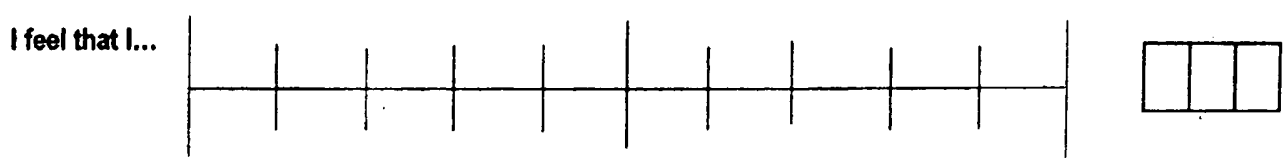

0

Definitely

Would Use

Definitely

Would Not Use
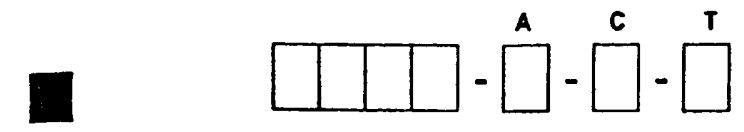
APPENDIX 3 - Hispanic Ethnicity/Identity Measure 


\section{ETHNIC ORIENTATION - HISPANIC}

1. Here are some questions about you and your ethnic group. People may describe their ethnicity in many different ways. How would you describe your ethnic identity?

$\begin{array}{ll}\square \text { Cuban } & \square \text { Mexican-American } \\ \square \text { Colombian } & \square \text { Dominican-American } \\ \square \text { Mexican } & \square \text { Nicaraguan-American } \\ \square \text { Dominican } & \square \text { Hispanic-American } \\ \square \text { Nicaraguan } & \square \text { Hispanic } \\ \square \text { American } & \square \text { LatinolLatina } \\ \square \text { Cuban-American } & \square \text { Other: }\end{array}$

Colombian-American

We are interested in your Ethnic Identity. By ethnic identity we mean the importance you attach to the personal identity you just described. Please use the numbers to indicate your level of agreement with each statement I read.

2. You have a strong sense of yourself as a member of your ethnic group.

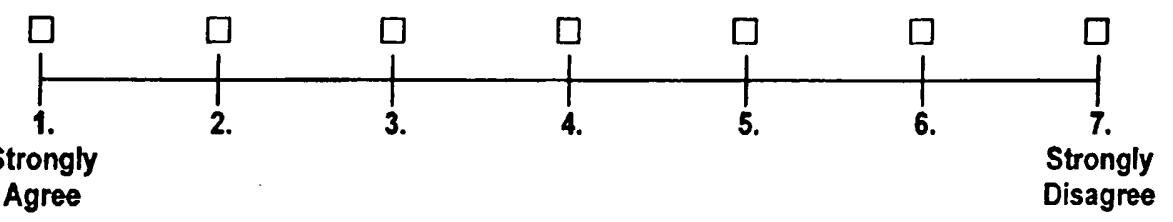

3. You identify with other people from your ethnic group.

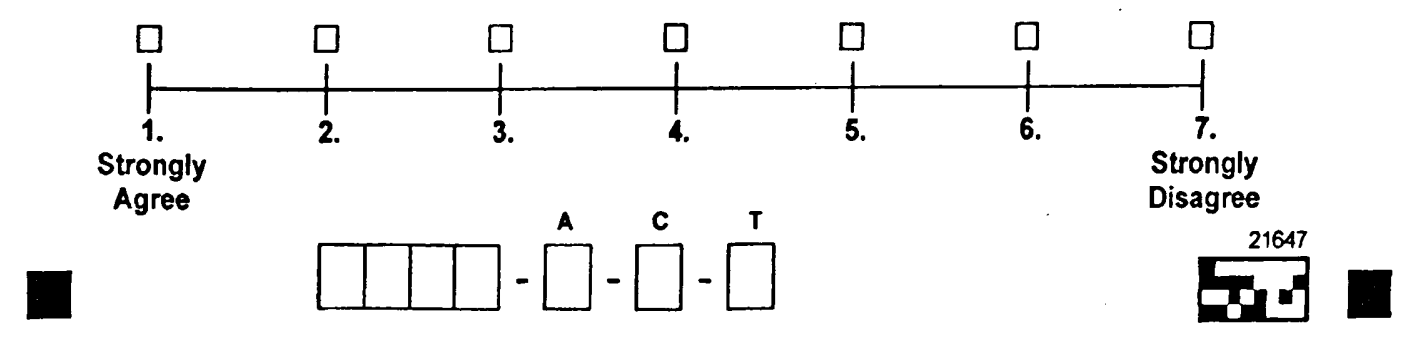


4. Most of your close friends are from your own ethnic group.

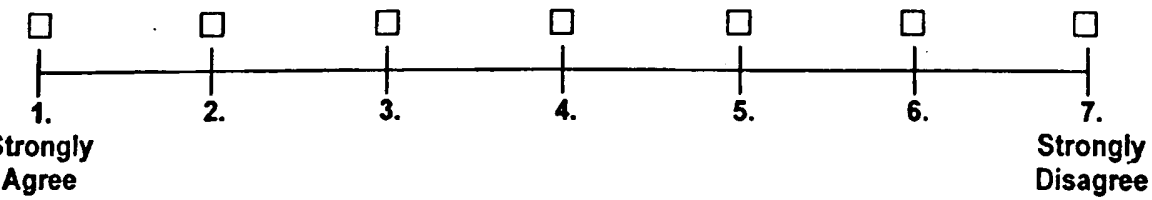

5. Your ethnic heritage is important in your life.

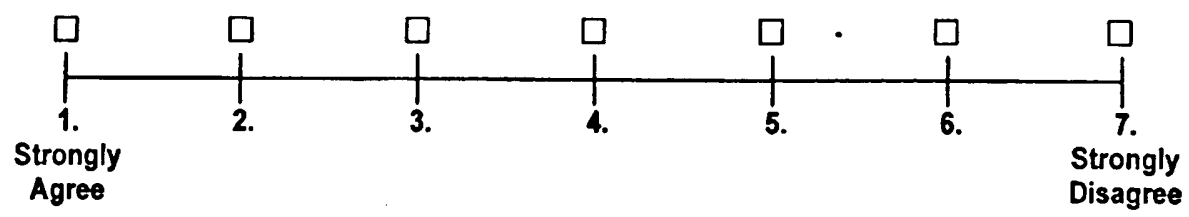

6. You are more comfortable in social situations where others are present from your ethnic group.

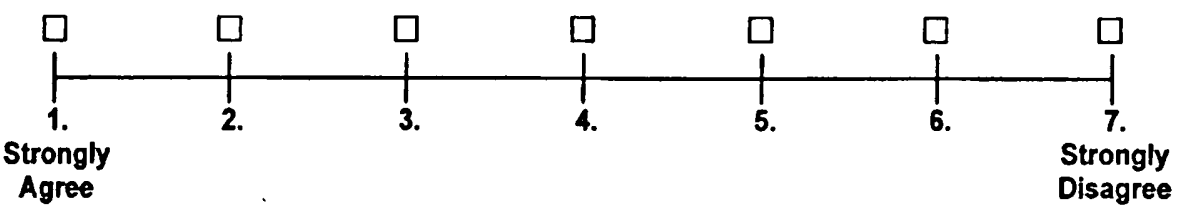

7. You are proud of your ethnic heritage.

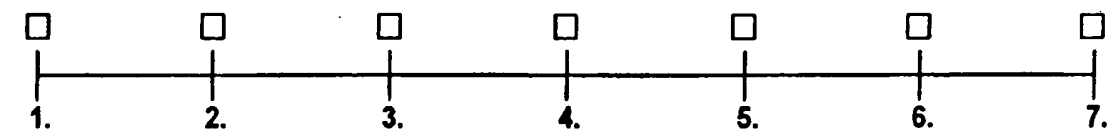

Strongly

Agree

Strongly

Disagree

8. Your ethnic group had a lot to do with who you are today.

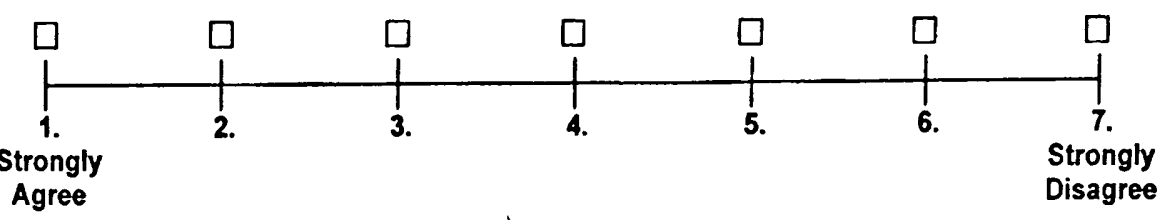

Agree
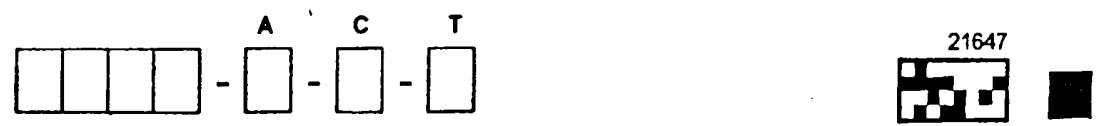
9. Your ethnic background plays a big part in how you interact with others.

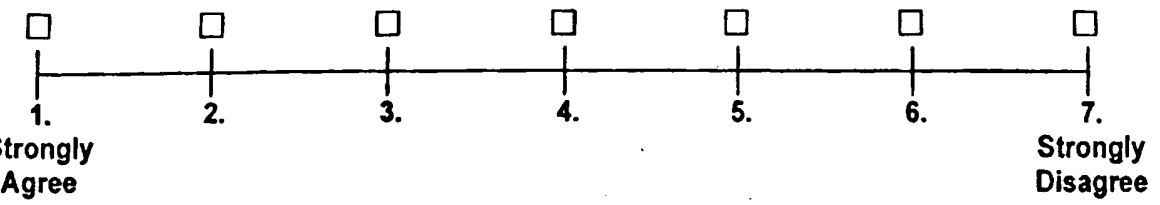

10. You prefer to date people from your ethnic group.

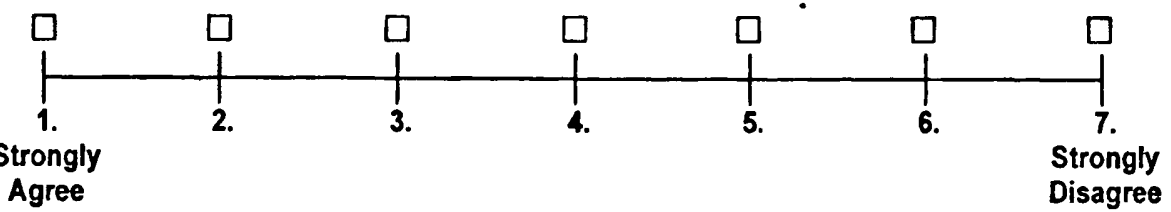

11. Your values, attitudes and behaviors are shared by most members of your ethnic group.

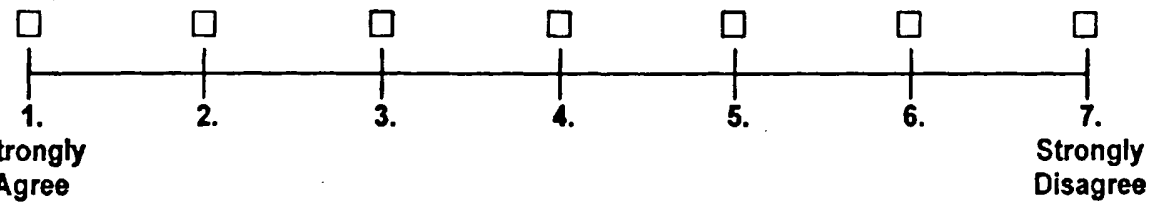

The next three questions concern treatment you may or may not have received from others because you are Hispanic (Latin). Please use the scale to indicate the answer that most closely reflects how often these things have happened to you.

12. How often do people dislike you because you are Hispanic (Latin)?

13. How often are you treated unfairly because you are Hispanic (Latin)?

14. How often have you seen friends treated badly because they are Hispanic (Latin)?
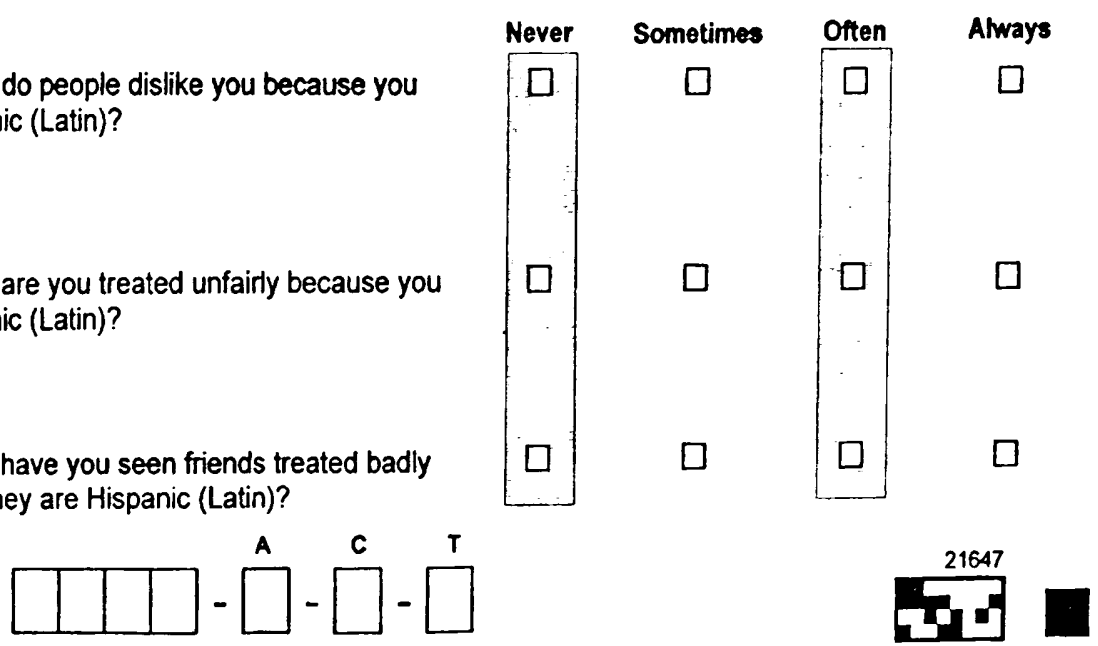
This next section deals with your language-related preferences. Please use the scale below to answer these questions.

Spanish
all the
time

$\begin{array}{ll}\text { Spanish } & \text { Spanish and } \\ \text { most of } & \text { English } \\ \text { the time } & \text { equally }\end{array}$

English most of English all the $\square$
$\square$
$\vdots$
$\square$
$\square$
$\square$
$\square$
$\square$
$\square$
$\square$
$\square$

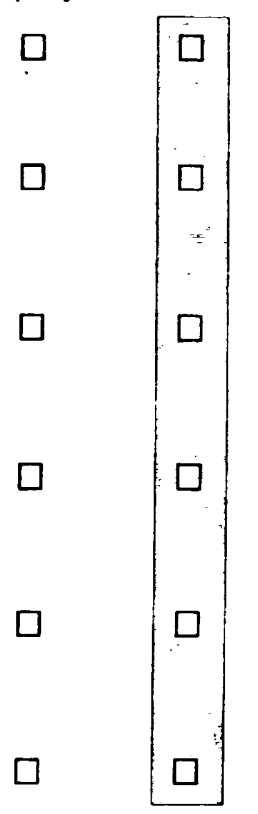

15. What language do you prefer to speak?

16. What language do (did) you speak at school?

17. What language do you speak with friends?

18. What language do you speak at home with your family?

19. In what language are the magazines you read?

20. In general, in what language are the movies, T.V. and radio programs you like to watch and listen to the most?

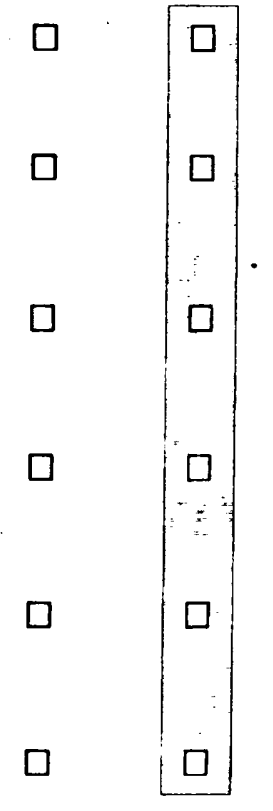

$$
\text { to watch and listen to the most? }
$$

$$
\text { . }
$$

Please answer the next two questions independently of one another. Use the scale below to indicate your level of agreement with each statement.
22. You consider yourself to be American.
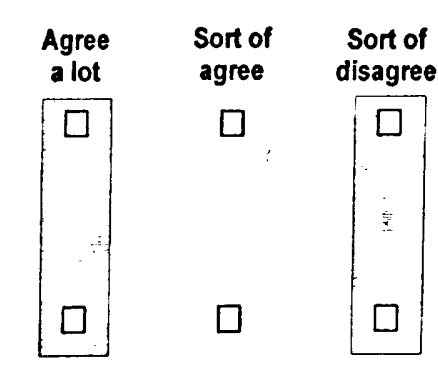
21. You consider yourself to be Hispanic or Latino(a).

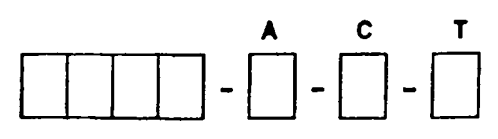

